\title{
THEORY OF UNIVERSALITY
}

\author{
Kasibhatla Surya Narayana ${ }^{1}$ \\ (Material Department, HMT Machine Tools Ltd, Bangalore-560013, India )
}

\section{ABSTRACT :}

This theory is an attempt to describe the universal phenomena like space, time, matter and energy as an inter-relationship bound by a newly discovered force named as the universal force. The universal force is shown to be the force of gravitation, electricity, magnetism, strong nuclear and weak nuclear forces. I believe any other force, hitherto fore not discovered; also, can be explained in terms of this universal force.

Liberal use of wave-particle duality, relativity, quantum concepts is made to achieve a harmonious and comprehensive synthesis of all the existing beliefs in physics into a new theory with some new concepts added here and there. While adding new concepts, enormous care has been taken to ensure that the existing beliefs are not contradicted. Moreover, the new concepts are proved to be correct theoretically by deriving the constants like $G, \sigma$, etc.,

\section{Keywords : Space, Time, Matter, Energy, Universal Force}

1. Email : ksuraxn@yahoo.co.in 


\section{CONTENTS}

1. INTRODUCTION

2. POSTULATES

3. INDENTATIONS AND EQUATIONS

4. PROOF

5. CONCLUSIONS

6. ANNEXURE - I

7. ANNEXURE - II

8. ANNEXURE - III

9. ACKNOWLEDGEMENT

10. REFERENCES 


\section{INTRODUCTION}

\section{UNIVERSE \& UNIVERSALITY}

From time immemorial, the struggle to understand the phenomena surrounding us, has been our chief occupation, second only to the struggle to survive. After successfully surviving the predators and harmful wild creatures, we have started building secure measures for food, cloth and shelter. Nevertheless, the natural calamities like floods, fire, earthquakes, and disease continue to wreak havoc with all the life and human life in particular. Death and disease could not be overcome fully by all the scientific measures invented so far.

We have started exploring our immediate environment in an attempt to understand the natural phenomena surrounding us. This attempt to understand is only to build safety and survival from the onslaught of the Nature's fury and to harness Nature's forces to the benefit of humankind. In the process, various gods and goddesses are proposed to be the cause of various phenomena. Sometimes, sacrifices are offered to gain the favor of the deities and worship also is done to please the gods and goddesses. For example, many rituals are done to get timely rain and to reap a good harvest. Worship is done for timely sunrise also. Slowly, we have realized the Sun and the Moon follow a regular pattern of rise and set whether a worship is done or not, and whether a sacrifice is made or not. We also, came to know that the celestial bodies behave in a regular pattern. Hence, the proposal came that the Earth was the centre of the world and the celestial bodies went around the Earth according to this pattern. Later investigations revealed that the Earth and the planets are spherical bodies which went around the Sun. Further, it was revealed by Newton's gravity and confirmed by Einstein's' relativity that gravity is indeed the major force acting among the celestial objects including our solar system.

Moreover, we have generalized the natural phenomena in the form of laws; because, they seem to follow the same behavior wherever and whenever we observe. Based on this observation, certain theories are proposed as the explanation for the behavior of the phenomena on the Earth and with regard to the celestial objects. A theory is a set of assumptions and rules, which describes the way the celestial bodies behave including the Earth on which we live. If the predictions made by the theory are observed in practice and also, if 
the theory can answer all the related questions, then, that theory is deemed as valid and used for further understanding of the universe.

As time progresses, better and more refined theories of the universal behavior are put forth for accurate, complete and comprehensive understanding of the universe we live in. Our ultimate goal is a total understanding of the universe. With the help of such understanding we can protect our life along with Solar System and Milky Way Galaxy against a possible, natural, cosmic calamity. So far, our assumption about the universe is that the laws which are valid on the Earth and the Solar System are also universally valid. This universality of the laws is at the basic core of the 'Theory of Universality' developed by the author 


\section{THEORY OF UNIVERSALITY}

This theory is an attempt to describe the universal phenomena, the interrelationships among the four fundamental quantities space, time, matter and energy with a precise and accurate mathematical quantification of the relationships. In the process, a force, named as universal force pervading the universe; and, which in turn manifests as every other force locally or universally present is also described. That is to say there is only one force, the universal force. Every/any other force is a manifestation of universal force.

Liberal use of Einstein's relativity, deBroglie's wave particle duality. Planck's quantum radiation is used to synthesize all the existing beliefs into a harmonious and comprehensive " Theory of Universality".

\section{POSTULATES}

1. Space is the medium using which light travels.

2. Speed of light is the critical attainable speed in our universe.

3. The universe when the projection of time $\left(4^{\text {th }}\right.$ dimension) is taken on a three dimensional medium, is like the two-dimensional sheet (shadow) model of a three - dimensional object in 2-dimensions.

4. Nothing in the universe can be fully destroyed.

5. Space, Time, Matter and Energy are inter-related. They exist together in anywhere and everywhere in the universe. None of the four fundamental quantities like space, time, matter and energy is uniquely existent in the universe.

6. There exists a very powerful force to be named as the universal force, because of which other forces like gravitation, electromagnetism, strong and weak nuclear forces manifest.

7. The universal force condenses space into matter.

8. Every physical law has some inertia of existence which is quantifiable in space-time-matter-energy frame of the universe.

9. Any physical law can be created and preserved with a certain inertia in space-time-matter-energy frame. 
10. The truth of the universe keeps changing depending on where, how and at what time the observation for a certain truth is made with an inclination to reach experimental proof of theoretical conjectures.

In all, there are ten vital formulae; six of them describe the relationship among space, time, matter and energy; four of them describe the relationship between universal force and space, time, matter and energy.

\section{INDENTATIONS AND EQUATIONS}

Assume that there is a particle of mass $m$, Compton wave length $\ell$; let, the energy stored in it be $\mathrm{E}$. Assume, the universal force utilized in creating the particle is $F$. Here, let me assert that the universal force which created the particle also bestows on it a time of ' $t$ ' , $t$ is the time of inertia of existence the particle will have during which its changes become nil. Any change anywhere in the universe manifests as time. The time inertia (also called laziness of the particle) between two successive changes is ' $t$ '. Then the 10 (ten) formulae giving the relationship among space, time, matter and energy and $F$ are as follows:

$1 \mathrm{E}=m \mathrm{c}^{2}$ Einstein's mass-energy equivalence

2. $\ell=h / m c$ space-mass equivalence

3. $\mathrm{t}=\mathrm{h} / \mathrm{mc}^{2}$ mass-time equivalence

4. $E=h c / \ell$. Energy-space equivalence

5. $\mathrm{E}=\mathrm{h} / \mathrm{t}$ Energy-time equivalence

6. $\ell=c t$ space-time equivalence

7. $\mathrm{F}=\mathrm{hc} / \ell^{2}$ Force-space equivalence

8. $\mathrm{F}=\mathrm{h} / \mathrm{C} \mathrm{t}^{2}$ Force-time equivalence

9. $F=E^{2} / c h$ Force-energy equivalence

10. $F=m^{2} c^{3} / h$ Force-mass equivalence

Where $\mathrm{h}=$ Planck's constant ; $\mathrm{c}=$ speed of light Derivation of the equations:

1. $E=m c^{2}$ is the popularly already- known equation of energy-mass equivalence of Einstein's 


\section{$2 \cdot \ell=h / m c$}

\section{Einstein}
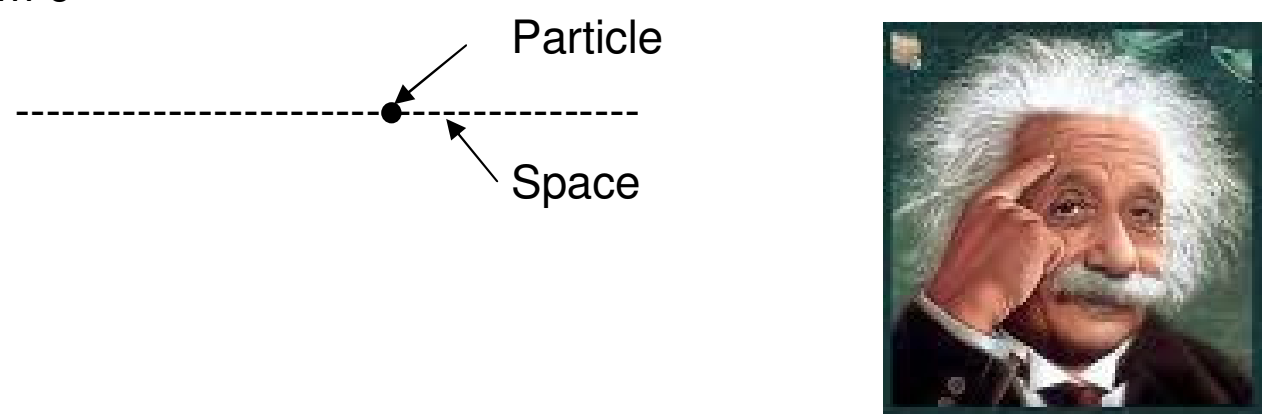

Space, is like a sheet in the $4^{\text {th }}$ dimension of time, That is, assuming we have entered the $4^{\text {th }}$ dimension of time, and, we are able to perceive past, present, and future as one; then, the 3 dimensional space, would look like 2-dimensional shadow in the time frame. The universal force $F$ condenses space into matter. Suppose the energy spent / created in creating a particle of mass $m$ is $E$. Suppose, the Compton wave length of the particle is $\ell$, with a time inertia (also called laziness of the particle) of $t$. The mass - space vibration within the particle moves at a speed c, speed of light, to achieve simultaneity every where. Then, by wave - particle duality (of Louis d'Broglie):

$$
\begin{aligned}
& \ell=\mathrm{h} / \mathrm{m} \text { c- this is true in case of proton. } \\
& \mathrm{m}=\text { rest mass of neutron or proton }=1.674 \times 10^{-27} \\
& \mathrm{~h}=\text { Planck's constant }=6.626 \times 10^{-34} \\
& \ell=\text { proton Compton wavelength works out to be } 1.319 \times 10^{-15}
\end{aligned}
$$

by above equation of $\ell=\mathrm{h} / \mathrm{m}$ c; which agrees with the experimental value of $1.321441 \times 10^{-15}$-- proved! (all in MKS units). Similarly, in the case of an electron; $\ell=\mathrm{h} / \mathrm{m}$ c.

$\mathrm{m}=9.109 \times 10^{-31} \mathrm{c}=3 \times 10^{8} \mathrm{~h}=6.626 \times 10^{-34}$

$\ell$ works out to be $2.424 \times 10^{-12}$ which is Compton wavelength of an electron which agrees with experimental result of $2.4263107 \times 10^{-12}$ - proved !

So, the equation $\ell=h / m c$; where $\ell$ is Compton wavelength ; $\mathrm{m}$ is the rest mass is true in the case of proton / electron.

$$
3 \cdot \mathrm{t}=\mathrm{h} / \mathrm{m} \mathrm{c}^{2}
$$

By Planck's principle of Quantum radiation of energy; this very particle behaves like an energy packet of wavelength $\ell$ ( $\ell$ is the Compton wave length ) and time t. 
$E=m c^{2}=h v=h / t \quad \rightarrow t=h / m c^{2}$

This implies that for an electron $\mathrm{m}=9.109 \times 10^{-31} \mathrm{~kg}$;

$t=8.081 \times 10^{-21}$ ( all in MKS units everywhere) is the laziness or time inertia. This means that between two successive changes to an electron; there must necessarily be a period of $8.081 \times 10^{-21}$ seconds. After which period only, a change can be enforced on an electron. So, it is impossible to change an electron in position or pace or state faster than this. If so, it will no longer be electron.

4. $E=h c / \ell$. We already know that $E=h \vee$ (Planck's theory). But, $v=c /$ $\ell, \quad v$ is the frequency of the energy packet contained in the particle. $\rightarrow \mathrm{E}$ $=\mathrm{h} \mathrm{c} / \mathrm{l}$

5. $E=h / t$, .This is simple; because, $E=h v$; and $v=1 / t \rightarrow E=h / t$.

Note: it is assumed that a particle of time inertia t., will also behave like a wave of cycle time ' $t$ '. ., when converted into energy $E$. therefore, $E=$ $\mathrm{h} / \mathrm{t}$.

6. $\ell=c$ t. Now, the particle has mass ' $m$ ' in all directions of observation, and points of observation within the particle. This is possible only when the mass-space vibration within the particle moves at the speed of light (to ensure simultaneity) of wavelength $\ell$.(compton wave length)

$\ell / \mathrm{t}=\mathrm{c} \rightarrow \ell=\mathrm{ct}$

7. $\mathrm{F}=\mathrm{h} \mathrm{c} / \ell^{2}-$ when universal force $\mathrm{F}$; acts on the particle to create a particle of Compton wave length $\ell$; the energy spent / created is;

$\mathrm{F} . \ell=\mathrm{h} v=\mathrm{hc} / \ell \rightarrow \mathrm{F}=\mathrm{hc} / \ell^{2}$

A force of universal force $=3.395 \times 10^{-2}$ Newtons is spent in creating the particle electron.

8. $\mathrm{F}=\mathrm{h} / \mathrm{Ct}^{2}$.It is assumed that the time inertia of a particle is the same as its cycle time when converted into energy-wave. This means $\ell=\mathrm{ct}$; applied to the equation -7 gives:

$\mathrm{F}=\mathrm{h} \mathrm{c} / \mathrm{l}^{2}=\mathrm{hc} / \mathrm{c}^{2} \mathrm{t}^{2}=\mathrm{h} / \mathrm{ct} \mathrm{t}^{2} . \rightarrow \mathrm{F}=\mathrm{h} / \mathrm{ct} \mathrm{t}^{2}$ 
9. $F=E^{2} / c h$. Energy spent in creating a particle of compton wave length $\ell$ , from the basics of physics is $E=F$. $\ell$. Therefore, $F=E^{2} / c h$

10. $F=m^{2} c^{3} / h$. from equation -9 , we have $F=E^{2} / c h$. We know that, $E$ $=\mathrm{m} \mathrm{c}^{2}$. therefore, $\mathrm{F}=\mathrm{m}^{2} \mathrm{c}^{3} / \mathrm{h}$.

\section{PROOF}

Proof that my theory is correct and valid follows. I will theoretically derive the value of ' $G$ ' (gravitational constant) and show that this coincides with experimental value.

Consider the gravitational constant as $\mathrm{G}$, and take the gravitational force between the Sun and Earth as F. We know that

$$
F=\frac{G M_{1} M_{2}}{R^{2}}
$$

$$
\text { where } \begin{aligned}
& M_{1}=\text { mass of Sun } \\
& M_{2}=\text { mass of Earth } \\
& R=\text { mean distance between the } \\
& \text { Earth and the Sun }
\end{aligned}
$$

But, according to my theory of universality, the universal force exerts a force of $\mathrm{m}^{2} \mathrm{c}^{3} / \mathrm{h}$ for the particle. In the case of Sun, this universal force of the various atoms is compensated by various layers of the particles within the Sun; except the upper - most layer. In the case of upper-most layer half of the tension created by the universal force on space appears as gravitation towards the outer space away from the Sun, the other half is turned inwards...... and is duly compensated within. No. of atoms (mostly Hydrogen) on the outermost layer of Sun is

\section{$\frac{4 \pi R_{1}{ }^{2}}{4 \pi r_{1}{ }^{2}} \quad: \quad \begin{aligned} & \text { where the } R_{1} \text { is the outermost layer's radius on Sun } \\ & \text { and } r_{1} \text { is the mean free path of the Hydrogen atom on the }\end{aligned}$ Sun's surface}

The comprehensive force released by this on the outer space away from the sun is :

$\frac{4 \pi R_{1}^{2}}{4 \pi r_{1}^{2}} \quad \frac{m^{2} c^{3}}{h} \cdot 1 / 2:$ the factor $1 / 2$ appears because the other half is exerted by the Sun is : 


$$
1 / 2 \cdot \frac{4 \pi R_{1}^{2}}{4 \pi r_{1}^{2}} \cdot \frac{m^{2} c^{3}}{h}
$$

The force experienced by the Earth due to the force emanating from the Sun is

$$
\frac{4 \pi R_{2}^{2}}{4 \pi R^{2}}
$$

fraction of the total force $: \Rightarrow F=G \cdot \frac{M_{1} M_{2}}{R^{2}}=1 / 2 \cdot \frac{4 \pi R_{1}{ }^{2}}{4 \pi r_{1}{ }^{2}} \cdot \frac{m^{2} c^{3}}{h} \cdot \frac{4 \pi R_{2}{ }^{2}}{4 \pi R^{2}}$

Where $R_{2}$ is the radius of the Earth; $R$ is mean distance between the Sun and the Earth. The fraction only is experienced because that is the area covered by the Earth. If the total area of $4 \pi R^{2}$ from the sphere encompassing the Earth is considered. Again, half of this is not considered because, this is fully experienced by the Earth and therefore, this is the gravitational force exerted by the Sun and experienced by the Earth from the Sun. (Actually, volume and density should be taken. As an approximation area is taken)

Note: all values are taken in MKS units everywhere.

$$
\Rightarrow G=\frac{R^{2}}{M_{1} M_{2}} \cdot 1 / 2 \cdot \frac{4 \pi R_{2}^{2}}{4 \pi R^{2}} \cdot \frac{m^{2} c^{3}}{h} \cdot \frac{4 \pi R_{1}{ }^{2}}{4 \pi r_{1}{ }^{2}}
$$

$\mathrm{r}_{1}=$ mean tree path of the Hydrogen atom on the Sun's surface @ nearly $6000^{\circ} \mathrm{K}$. Mean free path of air @ 288 $\mathrm{K}=6.6317 \times 10^{-8}$. So, for Hydrogen atom approximately at temperature of $6000^{\circ} \mathrm{K}$.

$$
r_{1}=6.6317 \times 10^{-8} \times \frac{6000}{288} \times \frac{28.986}{1}=4.14481 \times 10^{-5} \text { metres }
$$

( 28.986 is the average air weight : 1 is Hydrogen atomic weight on Sun's surface @ $6000^{\circ} \mathrm{K}$. Hydrogen in that environment is in atomic state and not in molecular state. It is assumed that mean free path is directly proportional to the temperature and inversely proportional to the atomic weight.) .

In the derivation of the gravitational constant $\mathrm{G}$, the factor 1 was taken for the universal force on the outer space atoms of Hydrogen on SUN ; because, this factor $1=$ atomic weight of Hydrogen atom for proton was taken in the derivation of the mean free path for Hydrogen atom on the sun's surface ( atmosphere) @ $6000^{\circ} \mathrm{K}$, because the Hydrogen is in atomic state and not in molecular state. 
$r_{1}=$ mean free path of Hydrogen on sun's Surface $=4.14481 \times 10^{-5}$

$\mathrm{R}_{2}=$ Radius of Earth $=6.371 \times 10^{6}$

$\mathrm{R}_{1}=$ Radius of Sun $=6.960 \times 10^{8}$

$\mathrm{m}=$ mass of the proton $=1.674 \times 10^{-27}$

c $=$ Speed of light $=3 \times 10^{8}$

$\mathrm{h}=$ Planck's constant $=6.626 \times 10^{-34}$

$\mathrm{M}_{1}=$ Mass of Sun $=1.99 \times 10^{30}$

$\mathrm{M}_{2}=$ Mass of Earth $=5.977 \times 10^{24}$

Substituting the above values for $r_{1}, R_{2}, R_{1}, m, c, h, M_{1}, M_{2}$ in

equation - A above;

we get $G=5.871 \times 10^{-11}$

which agrees with the experimental value of $6.673 \times 10^{-11}$.

The slight discrepancy can be due to the various assumptions and approximations and the too simple an approach taken for evaluating the mean free path of Hydrogen atom on the Sun's surface.

Hence, my theory is proved to be right !

Derivation of Stephen's constant : (MKS Units)

To prove my theory is right and valid once again :

Consider the total force emanating from the sun is

$$
F=1 / 2 \cdot \frac{R_{1}^{2}}{r_{1}^{2}} \cdot \frac{m^{2} c^{3}}{h}
$$

according to my theory.

Where $r_{1}=4.144181 \times 10^{-5}$ is the mean free path of Hydrogen on Sun's atmosphere.

But, according to my force - energy equivalence ,

$F=E^{2} / c h \Rightarrow E=\sqrt{F c h} \Rightarrow E=\sqrt{1 / 2 \frac{R_{1}^{2}}{r_{1}^{2}} \frac{m^{2} c^{3}}{h} c h}=\frac{1}{\sqrt{2}} \cdot \frac{R_{1}}{r_{1}} \cdot m c^{2}$

: because of this energy outpouring away from the Sun ; the maximum power emanating from the Sun is $E / t_{p}$ where $t_{p}$ is the time inertia ( or laziness of 
proton). $t_{p}$ is the minimum time required for releasing energy by Sun's outermost Hydrogen surface. Therefore, maximum power released by the sun is ,

$P=E / t_{p}=\frac{1}{\sqrt{2}} \cdot \frac{R_{1}}{r_{1}} \cdot \frac{m c^{2}}{t_{p}}$

where $t_{p}=h / \mathrm{m} \mathrm{c}^{2}$ according to my theory.

Hence $P=\frac{1}{\sqrt{2}} \cdot \frac{R_{1}}{r_{1}} \cdot \frac{m^{2} c^{4}}{h}$

But, according to Stefan-Boltzmann law of radiation : the total energy, E, of all wave lengths radiated per second per square meter by a full radiator at temperature $T$ to surroundings at temperature $T_{0}$ is given by $E=\sigma\left(T^{4}-T^{4}{ }_{0}\right)$ where $\sigma$ is Stefan's constant. The maximum power released by Sun (considered as full radiator) at any time is $\sigma\left(\mathrm{T}^{4}-\mathrm{T}^{4}{ }_{\mathrm{o}}\right) \times 4 \pi \mathrm{R}_{1}{ }^{2}$-.- C

where $4 \pi R_{1}^{2}$ is the total surface area of Sun.

Now, $\mathrm{T}_{0}=0^{0} \mathrm{~K}$ (approximately)

Therefore, from (B) \& (C) $\sigma=\frac{1}{4 \pi T^{4}} \cdot \frac{1}{\sqrt{2}} \cdot \frac{1}{r_{1} R_{1}} \frac{m^{2} c^{4}}{h}$

Substituting values for $T, R_{1}, r_{1}, m, c, h$; we get

$\sigma=5.314 \times 10^{-8} ;$ which agrees with the experimental value of

$\sigma=5.66969 \times 10^{-8}$, hence, my theory is RIGHT.

The slight discrepancy can be due to the approximations made in $T, T_{0}, r_{1}$

Values used are :

$$
\begin{aligned}
& T=\text { Surface temperature of Sun }=6000^{0} \mathrm{~K} \\
& T_{0}=\text { Temperature of surroundings }(\text { approximately }) 0^{0} \mathrm{~K} \\
& \mathrm{~m}=\text { mass of proton/ neutron }(\text { rest mass })=1.674 \times 10^{-27} \mathrm{~kg} \\
& \mathrm{c}=\text { speed of light }=3 \times 10^{8} \mathrm{R}_{1} \text { radius of sun }=6.960 \times 10^{8} \\
& \mathrm{r}_{1}=\text { mean free path of Hydrogen on sun's surface }=4.14481 \times 10^{-5} \\
& \mathrm{~h}=\text { Planck's constant }=6.626 \times 10^{-34}
\end{aligned}
$$




\section{Further Proof :}

Here, follows further proof that my theory is right and valid. I will now theoretically derive the values of $\in_{0}, \mu_{0}$, e and show that they coincide with the experimental results.

$$
\begin{aligned}
\epsilon_{0} & =\text { free space permitivity } \\
\mu_{\circ} & =\text { free space permeability } \\
e & =\text { elementary charge of electron/proton }
\end{aligned}
$$

First, derivation of $\in_{0}$

One more postulate in my theory:

Charge $e$, is same as mass $m_{e}$ of electron on its classical radius $r_{e}$. Electron's Compton wavelength is the wavelength it behaves (is observed) as a wave $\ell_{\mathrm{e}}$. Electron behaves like a mass of $m_{e}$ (rest mass), wavelength $\left(\ell_{e}\right)$ of Compton wavelength, laziness $\mathrm{t}_{\mathrm{e}}=\mathrm{h} / \mathrm{m}_{\mathrm{e}} \mathrm{C}^{2}$ and an energy packet of $\mathrm{m}_{\mathrm{e}} \mathrm{C}^{2}$, and a charge $e$ all at the same time and also like a particle. That is $e, m_{e}, r_{e}, \ell_{e}, E_{e}$ are all synonymous at the same time. What you get is what you are observing for. Energy due to electronic charge $=$

$$
\begin{gathered}
=\frac{\mathrm{e}^{2}}{4 \pi \epsilon_{0} \epsilon_{\mathrm{r}} \mathrm{e}}=\mathrm{m}_{\mathrm{e}} \mathrm{c}^{2} \text { ( mass - energy) } \\
\rightarrow \epsilon_{0}=\frac{1}{4 \pi} \frac{\mathrm{e}^{2}}{\mathrm{r}_{\mathrm{e}}} \cdot \frac{1}{\mathrm{~m}_{\mathrm{e}} \mathrm{c}^{2}}=\frac{1}{4 \pi} \cdot \frac{\left(1.602 \times 10^{-19}\right)^{2}}{2.817 \times 10^{-15}} \cdot \frac{1}{9.109 \times 10^{-31}} \cdot \frac{1}{9 \times 10^{16}} \\
\epsilon_{0}=8.842 \times 10^{-12} \mathrm{Fm}^{-1} \ldots \ldots \ldots . . \text { which agrees with experimental value } \\
\quad \text { of } 8.854 \times 10^{-12} \mathrm{Fm}^{-1} \ldots \ldots \ldots . \text { PROVED! }
\end{gathered}
$$

Which again proves that the assumption in the postulate is true and valid.

Now, to derive the value of $\mu_{0}$ ( free space permeability ):

We know; by using Ampere's law; the force of electron and proton as elements of current is $\left(\mu_{\circ} e^{2} c^{2}\right) / 4 \pi r^{2}$; where $r$ is the Hydrogen covalent radius; which means the mean distance between proton and electron (ie., 28pm). According to my theory of universality, the force (universal force)

due to a particle of mass $m$ is $\frac{m^{2} c^{3}}{h}$ 
the force due to proton in Hydrogen is $(m)^{2} c^{3} / h$ from the nucleus; the force experienced by electron in the orbit would be

$$
\frac{m^{2} c^{3}}{h} \cdot\left(4 / 3 \pi r_{e}^{3}\right) /\left(4 / 3 \pi r^{3}\right)
$$

Where $r_{e}$ is the classical radius of electron and $r$ is the covalent radius of Hydrogen. Therefore,

$$
\frac{\mu_{o} e^{2} c^{2}}{4 \pi r^{2}} \cdot \frac{m^{2} c^{3}}{h} \cdot \frac{r_{e}^{3}}{r^{3}}
$$

Substituting, the values for all except $\mu_{\circ}$; we get,

$\mu_{0}=1.0637748 \times 4 \pi \times 10^{-7}$ MKS units.

Which agrees with the experimental value of $4 \pi \times 10^{-7}$ for $\mu_{\circ}$

And $\mu_{r}$ is approximately 1 ( one) for Hydrogen. Hence, my theory is once again proved to be right and valid. Note: the universal force due to electron is considered negligible. Hydrogen in ICAO atmosphere is considered, because only then $\mu_{r}$ is 1 . Value of $e$ : Once again, consider the electronic charge energy ;

and equate it to mass-energy $\mathrm{m}_{\mathrm{e}} \mathrm{c}^{2} \cdot \mathrm{e}^{2} /\left(4 \pi \in_{0} \mathrm{r}_{\mathrm{e}}\right)$

According to my theory charge and mass are same at the same time

Substitute $\in_{0}=8.854 \times 10^{-12}$ in MKS units and we get $e=1.603 \times 10^{-19}$

Which agrees with experimental result of $1.602192 \times 10^{-19}$. I have derived $\mathrm{G}, \sigma$, $\epsilon_{0}, \mu_{0}$, using my theory and they have all agreed with experimental results. Hence, my theory is right and valid including the postulates made therein. In the derivation of $\mu_{0}$ Electron's classical radius and volume is considered ; because electron is taken as one continuous and cohesive particle ; in which case, area is not applicable.

Discoveries : ( PREDICTION - 1 ) :

1. I have made a discovery that the Earth is exerting a force on the sun which is nearly 20,000 billion times $\left(2 \times 10^{13}\right)$ the force which the Sun is exerting on the Earth. The force exerted by Sun on the Earth is manifesting mostly as gravitation. 
Force emanating from Sun and exerted on the Earth

$$
F_{1}=\frac{R_{1}{ }^{2}}{r_{1}{ }^{2}} \cdot \frac{m_{1}{ }^{2} c^{3}}{h} \cdot \frac{R_{2}{ }^{2}}{R^{2}} \cdot \frac{1}{2}
$$

Similarly emanating from Earth and exerted on the Sun

$F_{2}=\frac{R_{2}{ }^{2}}{r_{2}{ }^{2}} \cdot \frac{m_{2}{ }^{2} c^{3}}{h} \cdot \frac{R_{1}{ }^{2}}{R^{2}} \cdot \frac{1}{2}$

( according to my theory). $\quad \frac{F_{2}}{F_{1}}=\frac{m_{2}{ }^{2} r_{1}{ }^{2}}{m_{1}{ }^{2} r_{2}{ }^{2}}$

$\mathrm{m}_{1}=$ elementary particle mass of Hydrogen on Sun's surface.

$=1.674 \times 10^{-27}$

$r_{1}=$ mean- free path of Hydrogen on sun's surface $=4.14481 \times 10^{-5}$

Regarding the Earth's surface sans the atmosphere,

Nearly $75 \%$ is water ; $25 \%$ is sand which is mostly silicon

Silicon density $=2300$; water density $=1000$

Average surface density $=1325$

Silicon molecular weight $=28.09$

Water molecular weight $=18.02$

Average surface molecular radius $r_{2}$ is given by

$r_{2}{ }^{3}=\frac{(0.75 \times 18.02+28.09 \times 0.25) \times 1.674 \times 10^{-27}}{1325} \times \frac{3}{4 \pi}$

$r_{2}=1.83 \times 10^{-10}$ (Approximate)

$\mathrm{m}_{2} / \mathrm{m}_{1}=0.25 \times 28.09+0.75 \times 18.02=20.5375$

$F_{2} / F_{1}=2163.7258 \times 10^{10}$

Substituting $m_{1}, r_{1}, m_{2}, r_{2}$ in equation

(1) above; we get

$F_{2} / F_{1}=2163.7258 \times 10^{10}$

which is nearly 20,000 billion times ; or $2 \times 10^{13}$ times

The Earth exerts a force which is nearly 20,000 billion times ( the gravitational force of sun on the Earth ) on the sun. The sun is fusing with Hydrogen as the fuel. The enormous force ( nearly 20,000 billion times the sun's gravity on 
earth), which the Earth is exerting on the Sun helps Hydrogen to fuse and helps sun to remain a shining star.

2. Planets are drifting away from the sun . (REFER ANNEXURE - II)

The energy released by sun causes space of equivalent amount to be released at the same time. Because of this release of space, all planets are drifting away from the sun as given below.

$$
\begin{aligned}
& E=\frac{h c}{\ell} \Rightarrow \ell=\frac{h c}{E}=\frac{h c}{\sqrt{F c h}} ; \text { and } v \text { is the speed of drift of the space } \\
& {\left[t_{p}=\frac{h}{m c^{2}}\right] \Rightarrow v=\frac{m c^{3}}{\sqrt{F c h}}=\frac{m c^{3}}{\sqrt{2} \frac{R_{1}}{r_{1}} \mathrm{mc}^{2}}=\sqrt{2} \cdot \frac{r_{1}}{R_{1}} c \Rightarrow v=\sqrt{2} \frac{r_{1}}{R_{1}} c}
\end{aligned}
$$

Where $r_{1}$, is the mean free path of Hydrogen atom on the surface of the sun and $R_{1}$ is the radius of the sun. This is approximately $2.4382 \times 10^{-5} \mathrm{~m} / \mathrm{sec}$.

Which is approximately $768 \mathrm{~m} /$ year. $\mathrm{v}=768 \mathrm{~m} /$ year. This is the rate of Compton wave length of space released radially at the solar- corona,(solarcorona is considered; because inside this Solar Corona, the atmosphere interferes with space released)

\section{CONCLUSIONS}

1. The discrepancy in evaluating $G \& \sigma$ as compared to the experimental result is negligible.

2. It is possible to create any physical law and give it a certain amount of inertia in space - time - matter - energy frame.

3. It is possible to create planets, stars, satellites to the planets and it is equally possible to change and control the movement or momentum of any celestial body in the universe.

4. Reflection of light in a mirror is due to the nuclei gravity of the mirror. Incidentally, every neutron behaves like a black hole. The neutron gravity ( super gravity) bends light in a $U-$ turn. There is a time mirror on the surface of neutron because of which; the reflection ( image) looks equidistant from the mirror as the object.

5. Refraction of light is the bending of light by neutrons ( black holes ) within the atom . 
6. The black hole surface acts like a time mirror showing future as past.

7. Gravity nullification can be achieved for gravity - zero travel.

8. Every object in the universe displays time inertia ( also called laziness ) ; which is quantifiable as $t=h / m c^{2}$

9. Intelligence $=$ memory $X$ thinking ( a product ), whenever either becomes zero ; Intelligence becomes nil .

10. There is extra-terrestrial intelligent life.

11. There is time crunch in the universe as in the case of lightning. The same event is described by light faster than sound. Sound is not only coming after lightning is seen; but, also the thunder is heard for longer time than the time lightning is seen.

12. The laziness of any physical body is $\mathrm{t}=\mathrm{h} / \mathrm{mc}^{2}$; the duration during which no change can be enforced on the particle or body in any manner whatsoever.

13. Thought ( impulsive thought) is a gravity wave.

14. Cosmic waves can influence our intelligence, memory and thinking.

15. Earth moves or any celestial body moves in jerks and not in a continuous manner...... similar to Planck's hypothesis that energy moves in Quanta.

16. Any event is described by light, sound, and others over different durations of time.

17. Smell and taste travel like sound, energy, light etc.,

18. Thinking can be known in advance ( or at the same time) if we can sense gravity waves.

19. We can achieve vehicle - less travel other than walking, running, jumping, falling and crawling...Etc.,

20. We can predict certain events ( all, if required)

21. There is no perceptible boundary to the universe in space-time-matterenergy frame.

22. We can generate thinking in others along the pattern we desire using gravity waves. 
23. Time moves in a circle called circle of time. Any single point on it is present ; when we move in time by perception of changes around us ; we go into past and future at the same time from present. Any change anywhere manifests as time.

24. Time and passage of time are same.

25. Absolute time is maintained by black hole.

26. There is no zero time except on black hole.

27. All forces are components of the universal force ; manifestation of universal force

New Discovery: The Sun and the planets in our Solar System are going at a speed in the universe higher than the speed of light.

\section{Observations}

1. The Earth going round the Sun has a speed ( in an absolute sense ) higher than the speed of the Sun, whatever the speed of the Sun maybe. ( That is the Earth has its own speed plus the speed of the Solar System along with the speed of the Sun).

2. The starting mass of all celestial bodies is same.

3. A celestial body ( like the Earth ) going at a speed higher than the speed of the Sun has a mass less than the mass of the Sun. This is possible only if both are going at a speed higher than the speed of light as per equation.

$$
\mathrm{m}=\frac{\mathrm{m}_{0}}{\sqrt{1-\frac{\mathrm{v}^{2}}{\mathrm{c}^{2}}}}
$$

Conclusion: This means that we are in $a-v e$ World. The world of antimatter.

Incidentally there is a massive black hole in the Centre of the Universe around which the rest of the Universe is going around. The black hole is the window to the + v e world. 
Proof:

Assume:

$M=$ Mass of the Sun

$\mathrm{C}=$ Speed of light

$\mathrm{M}_{2}=$ Mass of mercury

$M_{1}=$ Mass of Venus

$\mathrm{T}_{2}=$ Period of Rotation of Mercury Round the Sun

$\mathrm{T}_{1}=$ Period of rotation of Venus round the Sun

$V_{2}=$ Speed of the planet mercury round the sun

$V_{1}=$ Speed of the planet Venus round the sun

$M_{0}=$ Starting mass of all the celestial bodies in the Solar system

$\mathrm{R}_{2}=$ Mean distance of mercury from the Sun

$\mathrm{R}_{1}=$ Mean distance of Venus from the sun

Then ,

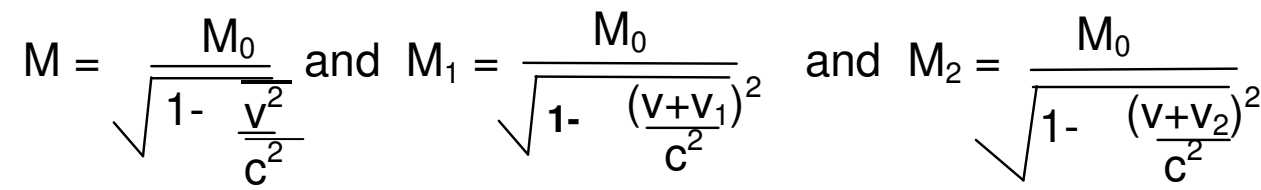

$\Rightarrow M \sqrt{1 \cdot \frac{v^{2}}{c^{2}}}=M_{1} \sqrt{1-\frac{\left(v+v_{1}\right)^{2}}{c^{2}}}=M_{2} \sqrt{1-\frac{\left(v+v_{2}\right)^{2}}{c^{2}}}$

$\Rightarrow\left(M^{2}-M_{1}^{2}\right) v^{2}-2 v v_{1} M_{1}^{2}-M_{1}^{2} v_{1}^{2}-c^{2}\left(M^{2}-M_{1}^{2}\right)=0$; we know that

$M^{2}>M_{1}^{2}$ ie., $M^{2}-M_{1}^{2}=M^{2}$

$\Rightarrow M^{2} \cdot v^{2}-2 v v_{1} M_{1}^{2}-\left(M_{1}^{2} v_{1}^{2}+c^{2} M^{2}\right)=0$; Approx

$\Rightarrow v=\frac{2 v_{1} M_{1}^{2} \pm \sqrt{4 v_{1}^{2} M_{1}^{4}+4 M^{2}\left(c^{2} M^{2}+M_{1}^{2} v_{1}^{2}\right)}}{2 M^{2}} ; c^{2} M^{2}>M_{1}^{2} v_{1}^{2}$

$\Rightarrow v=\frac{2 v_{1} M_{1}^{2} \pm \sqrt{4 v_{1}^{2} M_{1}^{4}+4 c^{2} M^{4}}}{2 M^{2}}$ Approx ; $c^{2} M^{4}>>v_{1}^{2} M_{1}^{4}$

$\Rightarrow v=v_{1} \frac{M_{1}^{2}}{M^{2}} \pm c=2.0993 \times 10^{-7} \pm c\left\{\begin{array}{l}v_{1}=0.35032 \times 10^{5} \mathrm{~m} / \mathrm{s}=\frac{2 \pi R_{1}}{T_{1}} \\ M=1.989 \times 10^{30} \mathrm{~kg} \\ M_{1}=4.869 \times 10^{24} \mathrm{~kg}\end{array}\right.$ 
Now, I will re-derive the Kepler's laws of planetary motion to prove that all the above assumptions are correct.

$M_{1}=\frac{M_{0}}{\sqrt{1-\frac{\left(v+v_{1}\right)^{2}}{c^{2}}}} ; M_{2}=\frac{M_{0}}{\sqrt{1-\left(\frac{v+v_{2}}{c^{2}}\right)^{2}}}$

$\Rightarrow M_{0}^{2}=M_{1}^{2}\left(1-\frac{\left(v+v_{1}\right)^{2}}{c^{2}}\right)=M_{2}^{2} \cdot\left(1-\frac{\left(v+v_{2}\right)^{2}}{c^{2}}\right)$

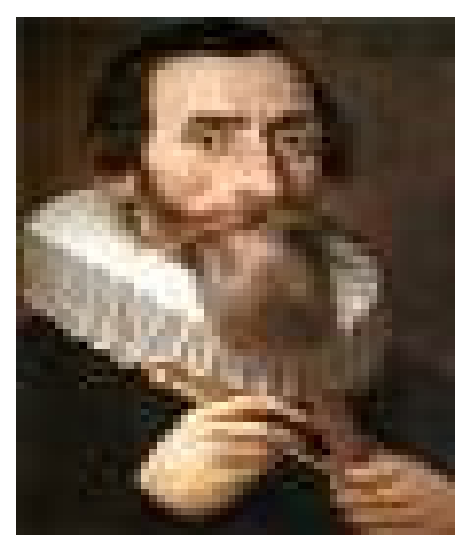

\section{Kepler}

$\Rightarrow M_{1}^{2}\left(2 v v_{1}+v_{1}^{2}\right)=M_{2}^{2} .\left(2 v v_{2}+v_{2}^{2}\right) ; v$ is very nearly equal to $c$ as per above ie., $\mathrm{v}=\mathrm{C}$

$\Rightarrow M_{1}^{2}\left(2 c v_{1}+v_{1}^{2}\right)=M_{2}^{2}\left(2 c v_{2}+v_{2}^{2}\right)$

Since $c$ is very much greater than $v_{1}$ and $v_{2}$

$M_{1}^{2} .2 c v_{1}=M_{2}^{2} .2 c v_{2}$

$\Rightarrow \mathrm{M}_{1}^{2} \mathrm{v}_{1}=\mathrm{M}_{2}^{2} \mathrm{v}_{2}$

$\Rightarrow \mathrm{M}_{1}^{2} \mathrm{v}_{1}=\mathrm{M}_{2}^{2} \mathrm{v}_{2}$

$\Rightarrow \quad \mathrm{M}_{1}^{2} \mathrm{v}_{1}=\mathrm{M}_{2}^{2} \mathrm{v}_{2} \Rightarrow \mathrm{M}_{\mathrm{i}}^{2} \mathrm{v}_{\mathrm{i}}=$ constant

In the case of planets $M_{i}^{2} v_{i}=$ constant where $M_{i}$ is the Mass of the planet and $v_{i}$ is its rotational speed around the sun.

The energy of a planet due to rotation around the sun is $1 / 2 M_{1} v_{1}^{2}$

The force is

$$
\frac{M_{1} v_{1}^{2}}{R_{1}}
$$

But, from my theory of Universality a force $F$ is equal to an energy $E$ given by $E$ $=$ F.ch $;$ where $\mathrm{h}=$ Planck's constant

This implies that $1 / 2 M_{1} v_{1}^{2}=\sqrt{\frac{M_{1} v_{1}^{2}}{R_{1}}}$ ch $\Rightarrow M_{1}^{2} v_{1}^{4}=\frac{4 M_{1} v_{1}^{2}}{R_{1}}$ ch $\Rightarrow M_{1}=\frac{4 c h}{R_{1} v_{1}^{2}}$

Substituting in A i.e., $M_{i}^{2} v_{i}=$ constant, we get 


$$
\frac{16 c^{2} h^{2}}{R_{1}^{2} v_{1}^{4}} \quad v_{1}=\text { constant } \Rightarrow \frac{16 c^{2} h^{2}}{R_{1}^{2} v_{1}^{3}}=\text { constant }
$$

But, we know that $v_{1}=\frac{2 \pi R_{1}}{T_{1}} ;$ where $T_{1}$ is the period of rotation around the sun, by the planet.

$\Rightarrow \frac{16 \mathrm{c}^{2} \mathrm{~h}^{2} \cdot \mathrm{T}_{1}{ }^{3}}{\mathrm{R}_{1}{ }^{2} \cdot 8 \pi^{3} \mathrm{R}_{1}{ }^{3}}=$ constant $\Rightarrow \frac{\mathrm{T}_{1}^{3}}{\mathrm{R}_{1}{ }^{5}}$ constant

By Kepler's laws of planetary motion:

1. Planets sweep equal areas in equal times $\frac{\pi R_{1}{ }^{2}}{T_{1}}=$ constant

2. The square of the periodic time is proportional to the cube of the mean distance of the planets from the Sun.

$$
\frac{\mathrm{R}_{1}^{3}}{\mathrm{~T}_{1}^{2}}=\text { constant }
$$

By equations (1) and (2) we get

$$
\frac{\pi \mathrm{R}_{1}{ }^{5}}{\mathrm{~T}_{1}{ }^{3}}=\text { Constant } \Rightarrow \frac{\mathrm{R}_{1}{ }^{5}}{\mathrm{~T}_{1}{ }^{3}}=\text { constant } \Rightarrow \frac{\mathrm{T}_{1}^{3}}{\mathrm{R}_{1}{ }^{5}}=\text { constant }
$$

Now, Both (B) \& (C) are the same.

Hence, Kepler's Laws are proved as per theory of Universality. This implies that the assumptions :

1. The planets and sun are traveling at a speed higher than the speed of light.

2. The starting mass of all celestial bodies is the same; are both correct and valid.

I have made a lot of approximations ( reasonable approximations ) in deriving the above. However, I believe the concept is more important than precision. 


\section{THEORY OF UNIVERSALITY}

Annexure - I

\section{Boltzmann Constant (k) :}

As per kinetic theory of matter; the molecules of an ideal gas at temperature (T) have a mean thermal kinetic energy given by $E=(3 / 2) k T$; in 3-dimensions where $\mathrm{k}$ is Boltzmann constant. However, in uni-directional consideration; the same is $E=1 / 2 k T$. The Sun's surface has mostly Hydrogen in atomic state. But, when hydrogen particles collide; they can fuse; the fusion dynamics being altogether different; let us take helium an inert gas; which does not fuse at the Sun's surface as reference. The layer below the Hydrogen layer is mostly Helium. Helium molecule has a mean free path given by

$r=6.6317 \times 10^{-8} \times(6000 / 288) \times(28.986 / 8)$

Where. $6.6317 \times 10^{-8}=$ mean free path of air @ $288^{\circ} \mathrm{K}$

$$
\begin{array}{ll}
6000^{\circ} \mathrm{K}= & \text { Temperature of the Sun's photosphere } \\
288^{\circ} \mathrm{K}= & \text { ICAO temperature of air. } \\
28.986= & \text { Average molecular weight of air. } \\
8 & =\text { molecular weight of Helium (two He } \\
& \text { monoatomic molecules) }
\end{array}
$$

Helium exists as molecules on Sun's surface; $r=0.5005897 \times 10^{-5}$

Now, suppose the Kinetic energy of Helium molecule is E; The Helium molecule travels in a linear path until collision. By my theory, $E$ is equal to a force $F$ given by $F=E^{2} / C h$. So, the Helium molecule travels with this force until collision, which is assumed perfectly elastic.

This implies: $F \cdot r=\left(E^{2} / c h\right) \cdot r=E \Rightarrow E=h c / r$.

Now, in one directional movement; which the Helium molecule does; till next collision:

$(1 / 2) k T=h c / r \Rightarrow k=(2 / T) \cdot(h c / r)=\frac{2}{6000} \cdot \frac{6.626 \times 10^{-34} \times 3 \times 10^{8}}{0.5005897 \times 10^{-5}}$

$\mathrm{k}=1.3236389 \times 10^{-23}$; which agrees with experimental value of

$k=1.38062 \times 10^{-23}$ 


\section{Hubble's Constant $H_{o}$ :}

Hubble's law is $v=H_{0} R$.

The Sun as per my theory is traveling at a speed $c+v$; where $v=2.0993 \times 10^{-7}$; light is the information carrier. With respect to the light (which is used to get information about the speed of separation of stars); the relative speed of separation of Sun is $v$. This is the speed of separation $(v)$ at the solar corona edge i.e.,21.14 $R_{s}$. (Approx). $R_{s}$ is the radius of Sun's photosphere. $=6.96 \times 10^{8}$ $\mathrm{m}$

Actually $R_{\mathrm{s} \text {-corona }}=21.14 \mathrm{R}_{\mathrm{s}}=147.1344 \times 10^{8} \mathrm{~m}$

Compton wavelength of Sun

$=2 \pi X R_{s-\text { corona }}$

The speed of Sun $c+v$ is present over its Compton wavelength.

$\Rightarrow \mathrm{H}_{0}=\frac{2.0993 \times 10^{-7}}{2 \pi \mathrm{X} 1.471344 \times 10^{10}}=2.2667 \times 10^{-18} \mathrm{~s}^{-1}$

agrees with experimental value of

$\Rightarrow \mathrm{H}_{0}=2.5 \times 10^{-18} \mathrm{~s}^{-1} \pm 15 \%$ found in

the year 2006 A.D.

Note: $R_{\text {s-corona }}$ is the radius of the Sun, till which atmosphere exists. (refer : Annexure -1 : Age of Sun since its birth )

\section{Strong Nuclear Force:}

Suppose, the Confinement radius is $R$

$$
\text { Quark - 1 Quark - 2 }
$$

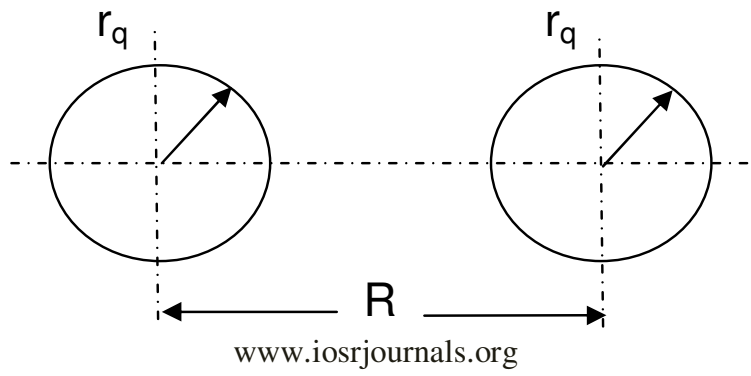




\section{Each, proton}

Comprises of three quarks

Quark mass $=m_{p} / 3=m_{q}$

$r_{q}=\left(r_{p} / 4\right) \times 1.732 ; r_{p}=\lambda_{p} / 2 \pi ; \lambda_{p}=$ proton Compton wave length.

$\epsilon_{\mathrm{r}} \cong 1$ for free space.

Then, the force of repulsion between two protons ( 6 quarks) is matched by the universal force of attraction as :

$$
\begin{gathered}
\frac{\left(6 \mathrm{~m}_{\mathrm{p}} / 3\right)^{2}}{\mathrm{c}^{3}} \frac{\frac{4 \pi}{\mathrm{h}} \times\left(\frac{\mathrm{r}_{\mathrm{p}}}{4} \times 1.732\right)^{3}}{\frac{4 \pi}{3}}=\frac{\mathrm{e}^{2}}{4 \pi \epsilon_{\mathrm{o}} \epsilon_{\mathrm{r}}} \frac{1}{\mathrm{R}^{2}} \\
\mathrm{R}=\frac{36 \times \mathrm{m}_{\mathrm{p}}{ }^{2} \mathrm{c}^{3}}{9 \mathrm{~h}}\left(\frac{\mathrm{r}_{\mathrm{p}}}{4} \times 1.732\right)^{3} \frac{4 \pi \epsilon_{\mathrm{o}} \epsilon_{\mathrm{r}}}{\mathrm{e}^{2}} \\
R=\frac{36 \times 1.672^{2} \times 10^{-54} \times 27 \times 10^{24}}{9 \times 6.626 \times 10^{-34}} \frac{\left(\frac{1.3214}{8 \pi} \times 1.732\right)^{3} \times 10^{-45} \times 4 \pi \times 8.854 \times 10^{-12}}{1.603^{2} \times 10^{-38}}
\end{gathered}
$$

$=1.4453 \times 10^{-15}$

which agrees with experimental result of $10^{-15} \mathrm{~m}$ (Approximately).

\section{Weak Nuclear Force :}

Energy at speeds greater than the speed of light 'c', behaves like anti-matter. Within radioactive neutron the energy trapped within the neutron behaves like anti-matter and splits neutron into proton and electron. The resultant repulsion between matter and antimatter separates proton and electron.

UF around a proton is

$$
\frac{m_{p}^{2} c^{3}}{h}
$$

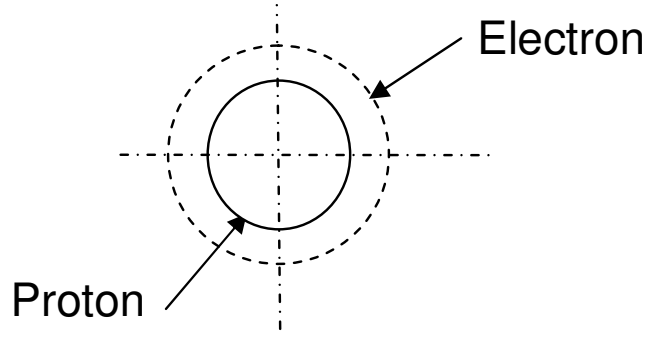


Electrostatic force is

$$
\frac{e^{2}}{4 \pi \in_{0} \in_{r} r^{2}}
$$

Where $r$ is the distance of separation between the two nearly concentric spheres of proton (radius $r_{p}$ ) and electron (radius $r_{e}$ ). Force is inversely proportional to the square of distance $r$ i.e., $r^{2}$. Energy is work done; a product of force and distance ( $r$ ). Therefore, energy is inversely proportional to the distance $r$ i.e.

$$
\frac{e^{2}}{4 \pi \epsilon_{o} r} \frac{r_{p}}{r_{e}} \text { is the energy in the creation of electron from a neutron }
$$

(against the attraction of proton on electron)and $r$ is the distance of the weak nuclear force by which proton and electron separate. This is equal to

the repulsion between matter and anti-matter $=\frac{m_{p}^{2} c^{3}}{h} \cdot r$

Therefore, $\frac{m_{p}^{2} c^{3}}{h} r=\frac{e^{2}}{4 \pi \epsilon_{o} r} \quad \frac{r_{p}}{r_{e}} \quad ; \epsilon_{r}=1$

$$
r=\frac{e^{2}}{4 \pi \epsilon_{o}} r \frac{h}{m_{p}^{2} c^{3}} \frac{r_{p} ;}{r_{e} ;} \frac{r_{p}}{r_{e}}=\frac{1.321 \times 10^{-15} X \frac{1}{2 \pi}}{2.426 \times 10^{-12} X \frac{1}{2 \pi}}
$$

$r^{2}=1.1023 \times 10^{-36}$

$r=1.0499 \times 10^{-18}$ which agrees with the experimental value of $10^{-18} \mathrm{~m}$

\section{Universal Gas Constant (R) :}

Take one mole of Hydrogen @ $273^{\circ} \mathrm{K}$. At this temperature Hydrogen is in molecular state. Therefore, mean free path of hydrogen is

$$
\begin{aligned}
& r_{H}=\frac{28.966}{1.00792 \times 2} \cdot \frac{6.6317 \times 10^{-8} \times 273}{288.15}=0.90277 \times 10^{-6} \mathrm{~m} \\
& V=\frac{4 \pi}{3} r_{H}^{3} \cdot N_{A}=18.56725 \times 10^{5}
\end{aligned}
$$

Where $N_{A}$ is Avogadro constant. A force of $\frac{m^{2} c^{3}}{h}$ is present at the 
surface of the object with mass $m$ and this force is present over a Compton wave length of $\lambda$ and a sphere of radius $(\lambda / 2 \pi)$. Assume $R_{0}$ is the radius of one mole hydrogen sphere; and $\lambda$ is the Compton wavelength of Hydrogen Molecule.

Force ' $F$ ' present at $R_{0}$ is given by :

$$
\mathrm{F}=1 / 2 \frac{\mathrm{m}^{2} \mathrm{c}^{3}}{\mathrm{~h}} \quad \frac{4 \pi \mathrm{R}_{0}^{2}}{4 \pi \mathrm{r}_{\mathrm{H}}^{2}} \quad \frac{4 \pi\left(\frac{\lambda}{2 \pi}\right)^{2}}{4 \pi \mathrm{r}_{\mathrm{H}}^{2}}
$$

We know that pressure $\mathrm{P}$ is Force per unit area.

$$
\mathrm{P}=1 / 2 \quad \frac{\mathrm{m}^{2} \mathrm{c}^{3}}{\mathrm{~h}} \quad \frac{4 \pi \mathrm{R}_{0}^{2}}{4 \pi \mathrm{r}_{\mathrm{H}}^{2}} \quad \frac{4 \pi\left(\frac{\lambda}{2 \pi}\right)^{2}}{4 \pi \mathrm{r}_{\mathrm{H}}^{2}} \quad \frac{1}{4 \pi \mathrm{R}_{0}^{2}}
$$

Also, $\mathrm{PV}=\mathrm{nRT}$

$$
\begin{gathered}
\Rightarrow R=1 / 2 \frac{\mathrm{m}^{2} \mathrm{c}^{3}}{\mathrm{~h}} \cdot \frac{\mathrm{R}_{0}{ }^{2}}{\mathrm{r}_{\mathrm{H}}{ }^{2}} \cdot \frac{\lambda^{2}}{4 \pi^{2} \mathrm{r}_{\mathrm{H}}{ }^{2}} \cdot \frac{1}{4 \pi \mathrm{R}_{0}{ }^{2}} \cdot \frac{\mathrm{V}}{\mathrm{nT}} \\
=1 / 2 \cdot \frac{4 \mathrm{~m}_{\mathrm{p}}{ }^{2} \mathrm{c}}{\mathrm{h}} \cdot \frac{\lambda_{\mathrm{p}}{ }^{2}}{4 \pi^{2} \cdot \mathrm{r}_{\mathrm{H}}{ }^{4}} \cdot \frac{1}{4 \pi} \cdot \frac{\mathrm{V}}{\mathrm{nT}} ; \mathrm{n}=1 \\
=1 / 2 \frac{1.6726^{2} \times 10^{-54} \times 27 \times 10^{24}}{6.6262 \times 10^{-34}} \times \frac{1.3214^{2} \times 10^{-30}}{\pi^{2} \times 0.90277^{4} \times 10^{-24}} \times \frac{18.56725 \times 10^{5}}{4 \pi \times 273 \times 1}
\end{gathered}
$$

$=8.21179$ which agrees with experimental value of 8.3143

\section{Free Space Impedance $\left(Z_{o}\right)$ :}

A wave of wavelength $\lambda$ behaves like a spherical object of radius $(\lambda / 2 \pi) . e_{\text {, }}$ is the charge of light when seen as a particle.

$$
E=I_{p}{ }^{2} \cdot R_{0} t_{\ell}=\frac{h c}{\lambda}=\frac{e^{2}{ }_{\ell}}{4 \pi \cdot \in_{0} \frac{\lambda}{2 \pi}} \Rightarrow e_{\ell}{ }^{2}=2 \epsilon_{0} . h c
$$

But, we know $\mathrm{I}_{\mathrm{p}}{ }^{2}=\frac{\mathrm{e}_{\ell}{ }^{2}}{\mathrm{t}_{\ell}{ }^{2}} \quad$; where $\mathrm{e}_{\ell}$ is the charge of light when seen as a 
particle and $t_{\ell}$ is the time inertia of light as a wave particle; that is when seen as a charge particle.

Therefore,

$\mathrm{E}=\mathrm{I}_{\mathrm{p}}^{2} \cdot \mathrm{R}_{0} \mathrm{t}_{\ell}=\frac{\mathrm{e}_{\ell}{ }^{2}}{\mathrm{t}_{\ell}^{2}} \cdot \mathrm{R}_{0} \mathrm{t}_{\ell}=\frac{\mathrm{e}_{\ell}{ }^{2}}{\mathrm{t}_{\ell}} \cdot \mathrm{R}_{0}$

But as per Planck's hypothesis, light of wavelength $\lambda$ and time inertia of $t_{\ell}$ behaves like an energy packet of energy $h / t_{\ell}$. From ----- 2 , we have

$\Rightarrow \frac{\mathrm{h}}{\mathrm{t}_{1}}=\frac{\mathrm{e}^{2} \mathrm{l}_{\ell}}{\mathrm{t}_{\ell}} \cdot \mathrm{R}_{0}$

$R_{0}=\frac{h}{e^{2}{ }_{l}}=\frac{h}{2 \epsilon_{0} c h}=\frac{1}{2 \epsilon_{0} c} \quad$ from

$R_{0}$ due to resistance is $R_{0}=\frac{1}{2 \epsilon_{0} C}$

$I_{0}$ due to inductance is $I_{0}=\frac{\mu_{0} C}{2}$

So, $Z_{0}$ due to impedance is $=R_{0}+I_{0}=1 / 2\left(\frac{1}{\epsilon_{0} C}+\mu_{0} c\right)$

As per maxwell's equation $\mu_{0} \in_{0}=\frac{1}{c^{2}}$

$\Rightarrow Z_{0}=1 / 2\left(\frac{1}{\epsilon_{0} C}+\mu_{0} c\right)=1 / 2 \cdot\left(\frac{1}{\epsilon_{0} C}+\frac{1}{\epsilon_{0} C}\right)=\frac{1}{\epsilon_{0} C}$

$Z_{0}=1 / \epsilon_{o} C=376.73021$

which agrees with experimental value of $Z_{0}=376.7304$

Alternatively, since $\mu_{o} \in_{o}=\left(1 / c^{2}\right) ; Z_{o}=\mu_{o} c$.

7.Speed of Rotation of the Sun $\left(\omega_{s}\right)$ :

$R_{s}=$ radius of sun, $\omega_{s}$ speed of rotation of sun,

$v_{s}=$ speed of sun in absolute sense, $M_{u}=$ starting mass of sun

$R_{s} \omega_{s}=v_{s} ; \ell_{s}=\frac{h}{M_{s} R_{s} \omega_{s}} ; \ell_{s}=\frac{h}{M_{u} C} \quad$ as per my theory 
$\Rightarrow \frac{h}{M_{u} \cdot c}=\frac{h}{M_{s .} R_{s} \omega_{s}} \Rightarrow \omega_{s}=\frac{M_{u}}{M_{s .}} \quad \frac{c}{R_{s .}}$

since momentum is conserved.

We know that $M_{u}$ for the Sun $=7.441 \times 10^{22}$

Hence, $\omega_{\mathrm{s}}=7.441 \times 10^{22} \times 3 \times 10^{8}=1.61172 \times 10^{-8}$. $1.99 \times 10^{30} \times 6.96 \times 10^{8}$

Observed rotation is 11 years; actual rotation is 12 years for the sun spots

$\Rightarrow \omega_{\mathrm{s}}=\frac{2 \pi .}{\mathrm{T}_{\mathrm{s}}}=\frac{2 \pi .}{12 \times 365 \times 24 \times 3600}=1.66099 \times 10^{-8} \cdot \mathrm{B}$

Now, both $(A)$ and $(B)$ are the same.

Note :

The space - mass vibration within the particle (Sun) released from the central object is ' $c$ ', and rotates at the speed of 'c'.

$M_{u}=$ initial mass of the sun as released from the central object.

\section{Newton's Law :}

$\mathrm{m}=$ rest mass

$\mathrm{m}_{1}=$ mass at speed $\mathrm{v}$

$\mathrm{u}=$ initial speed

$\mathrm{v}=$ final speed

$\mathrm{a}=$ acceleration due to force $\mathrm{F}$.

$\mathrm{u}=0$

Speed

$v^{2}=u^{2}+2 a l \Rightarrow v^{2}=2 a l$

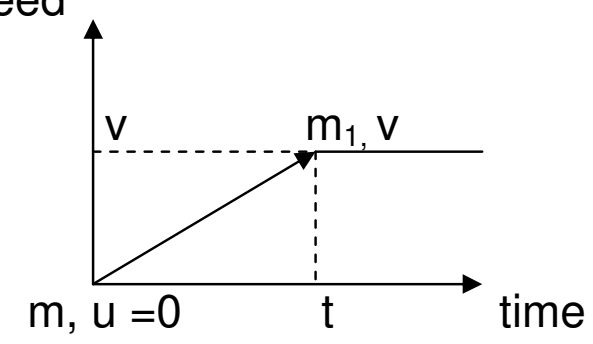

We know that as per my theory; $E=1 / 2 m_{1} v^{2}=\sqrt{\text { Fch }}$

$1 / 2 m_{1} v^{2}=\left(m_{1} / 2\right) 2 a \ell=m_{1} a \ell$

$\Rightarrow \sqrt{F c h}=m_{1} a \ell \Rightarrow F c h=m_{1}{ }^{2} a^{2} \ell^{2}$ 
But, according to my theory $\left(\mathrm{hc} / \ell^{2}\right)=\mathrm{F}$. A force $\mathrm{F}$ acting over a distance $\ell$ is equal to a force / energy wave of Compton wave length $\ell$.

Hence, Fch $=m_{1}{ }^{2} a^{2} .(h c / F) \Rightarrow F^{2}=m_{1}{ }^{2} a^{2}$

$\Rightarrow \mathrm{F}=\mathrm{m}_{1}$ a. Since at lower speeds $\mathrm{m}_{1} \approx \mathrm{m}$.

we have $\mathrm{F}=\mathrm{ma}$

$\ell, m_{1}, v, F$ are all applicable to force $F$. A force $F$ is equivalent to a Compton wave length of ' $\ell$ ' over which it is applicable. ' $\ell$ ' is here the distance over which the particle reaches speed ' $V$ ' from zero.

\section{CBMR - Cosmic Background Microwave Radiation :}

Assume CBMR is a radiation given out by the Earth.

$\mathrm{m}_{10}=$ rest mass of earth.

$\ell_{1}=$ wave length of the CBMR

$v_{1}=$ Speed of the earth around the Sun, which is going at the speed ' $c+v$ ' in absolute sense.

$\ell_{1}=$ wavelength of visible light.

$\mathrm{m}_{\mathrm{o}}=$ mass of light when treated as a particle.

By relativity $; \ell_{1}=\ell_{10} \cdot \sqrt{1-\frac{\left(c+v_{1}\right)^{2}}{c^{2}}}=\sqrt{\frac{2 v_{1}}{c}} \cdot \ell_{10}$

$$
t_{1}=t_{10} \cdot / \sqrt{1-\frac{\left(c+v_{1}\right)^{2}}{c^{2}}}=\sqrt{\frac{c}{2 v_{1}}} \cdot t_{10}
$$

$\frac{l_{1}}{t_{1}}=\frac{2 v_{1}}{c} \frac{l_{10}}{t_{10}}=2 v_{1}$; because $\frac{l_{10}}{t_{10}}=c$ as per theory

$V_{1}=\frac{2 v_{1}}{\ell_{1}}=\frac{2 v_{1}}{\ell_{\ell}}$ (in the visible light range $\quad \frac{\ell_{10}}{t_{10}}=c$

and $\frac{l_{1}}{\mathrm{t}_{1}}=\frac{\ell_{\ell}}{\mathrm{t}_{\ell}}$




$$
Y_{1}=\frac{2 \times 2.9818 \times 10^{4}}{404.7 \times 10^{-9}}
$$

is the peak frequency for the lowest wavelength of visible light i.e., violet light.

$=147.36 \mathrm{GHz}$ which agrees with experimental result of $160.4 \mathrm{GHz}$..

Note : $\mathrm{c}$ is the speed of light only; not that of microwave radiation nor that of ultra-violet radiation. Actually

$$
\begin{aligned}
& \mathrm{V}_{(\mathrm{IR})}<\mathrm{C} \\
& \mathrm{V}_{(\mathrm{UV})}>\mathrm{C}
\end{aligned}
$$

\section{Age of the Sun (since its birth) :}

Assumption : All celestial bodies jet out like a stream of particles from the central Black hole as particles; with the same starting mass and speed. $u$ is the external starting speed . $c$ is the space - mass vibration speed within the particle.

For all celestial bodies $\mathrm{M}_{\mathrm{i}}^{2} \mathrm{v}_{\mathrm{i}}=$ constant.

In the case of Sun $M_{v}{ }_{v}(c+v)=M_{u}^{2} \cdot u$; where $M_{u}=7.441 \times 10^{22}, M_{v}=1.99 \times 10^{30}$

$$
u=\left[\frac{1.99 \times 10^{30}}{7.441 \times 10^{22}}\right]^{2} \cdot c=2.1456 \times 10^{23}
$$

Time inertia of the primordial particle of Sun w.r.t. the central black hole Is $t_{u}=\frac{h}{M_{c}^{2}}$; one cycle time is $2 t_{u} \cdot \lambda_{u}$ w.r.t. the surroundings space is $\frac{h}{M_{u} u}$ ;the rate of expansion is $v_{\exp }=\frac{\lambda_{u}}{2 t_{u}}=\frac{c^{2}}{2 u}$ The sun expands to its present stage i.e., diameter of the Sun is total upto the edge of the solar corona, in time

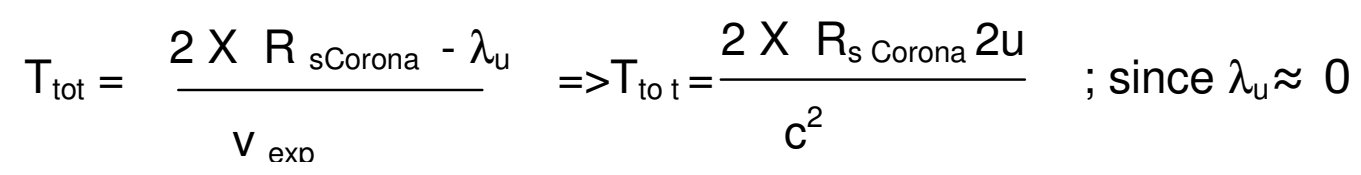




$$
=\frac{2 \times 21.14 \times 6.96 \times 10^{8} \times 2 \times 2.145610^{23}}{9 \times 10^{16}}=140.31 \times 10^{15}
$$

$=4.4491 \times 10^{9}$ years or 4.4491 billion years.

Which agrees with observed / calculated value of 4.5 billion years.

Note : Cycle time $=2 t_{u}$ is $t_{u}+t_{u}$ ie., the period of expansion + period of rest. The sun expands in jerks with total period of $2 \mathrm{t}_{\mathrm{u}}$.

$\mathrm{R}_{\text {Corona }}$ : Is the radius from the center of the Sun till the edge of the sphere till which Sun's atmosphere is present; and after which the Sun's atmosphere is negligible and ceases to exist.

The primordial particle of Sun slows down to the speed of

$C+v=C+2.0993 \times 10^{-7}$ to become Sun.

\section{DERIVATION OF R S-CORONA}

$\mathrm{R}$ geo, corona $=4 \mathrm{R} \in(\mathrm{R} \in$ is radius of earth $)$

g, geo, corona $=9.81 \times \quad \underline{R \epsilon^{2}}=0.613125$

$$
(4 \mathrm{R} \in)^{2}
$$

Similarly $R_{s}$, corona $=\sqrt{\frac{274}{0.613125}} \quad R s=21.14 R_{s}$

PREDICTION - 2 : The celestial objects jet out like bubbles from the central black hole, at speeds greater than the speed of light ; with VOID inside them; which helps them to float through space. The void does not have space, time, matter and energy and is perfectly empty.

The Geocorona of the Earth is a distance of $4 R_{E} \quad\left(R_{E}\right.$ is the radius of Earth ) from the center of the Earth., ie., $4 \mathrm{R}_{\mathrm{E}}$ is the space displaced by the Earth. This is equivalent to a space-mass of

$$
\frac{h}{4 R_{E . C}}
$$

By Archimedes Principle for floating objects this is the mass destroyed in creating the void for the Earth. Therefore, the Compton wave length of the void $\ell_{V}=2 \pi R_{V}=(h / c) /\left(\frac{h}{4 R_{E} . C}\right)=4 R_{E}$.

Hence, $\begin{aligned} \mathrm{R}_{\mathrm{V}} & =\text { radius of the void inside the Earth }=\frac{4 \mathrm{R}_{\mathrm{E} .}}{2 \pi}=\frac{2 \times 6371}{\pi} \\ & =4054 \mathrm{Km} .\end{aligned}$ 
This implies that the Earth's solid portion has a thickness of $R_{E}-R_{V}=6371-$ $4054=2317 \mathrm{Km}$ only. Except the top layer of crust and hydrosphere most of the solid material of the Earth consists of densest elements like OSMIUM or PLATINUM or IRIDIUM. 


\section{THEORY OF UNIVERSALITY}

Annexure - II

\section{Separation of Earth from Sun :}

The planet Earth is getting separated away from the Sun due to release of energy by the Sun (which in turn releases space which pushes the Earth away from the Sun)

The total force released by the Sun as per my theory is :

$$
\mathrm{F}=\frac{1}{2} \cdot \frac{\mathrm{R}_{1}^{2}}{\mathrm{r}_{1}^{2}} \cdot \frac{\mathrm{m}^{2} \mathrm{c}^{3}}{\mathrm{~h}}
$$

Energy out pouring : $\quad E=\sqrt{F c h}=\frac{1}{\sqrt{2}} \cdot \frac{R_{1}}{r_{1}} \cdot m c^{2}$

But, as per my theory this energy outpouring away from the sun releases space as given by

$$
\ell=\frac{h c}{E}=\sqrt{2} \cdot \frac{r_{1}}{R_{1}} \cdot \frac{h}{m c}
$$

This is the compton wave length of the space released :-

Therefore, the thickness of the space layer released is :

$$
r_{s}=\frac{\ell}{\pi}=\frac{\sqrt{2}}{\pi} \cdot \frac{r_{1}}{R_{1}} \cdot \frac{h}{m c}
$$

This increase in the space layer is felt at the solar corona $R_{S-c o r o n a}$ radially. Increase in space linearly along the plane of the Sun - Earth is (at the surface of Sun)

$$
r_{\mathrm{SE}}=\frac{\sqrt{2}}{\pi} \cdot \frac{\mathrm{r}_{1}}{\mathrm{R}_{1}} \cdot \frac{\mathrm{h}}{\mathrm{mc}} \cdot \frac{\pi \mathrm{R}_{1}{ }^{2}}{4 \pi \mathrm{R}_{1}{ }^{2}}
$$


Now the space - volume is conserved in the same way as mass- energy is conserved.

Increase in space linearly along the plane of the Sun - Earth @ the surface of Earth

$r_{E}=\frac{\sqrt{2}}{4 \pi} \quad \frac{r_{1}}{R_{1}} \quad \frac{h}{m c} \cdot \frac{\pi R_{2}^{2}}{4 \pi R_{2}^{2}} \cdot \frac{4 \pi R_{s}^{2} \text {-corona }}{4 \pi R^{2}}$

Where $R_{\text {s-corona }}=21.14 \times R_{1}=$ Radius of solar corona

$\mathrm{R}=$ Mean distance between the Sun and the Earth. The space is released at the least time period which is that of the time inertia of one

hydrogen atom on the Sun's surface $t_{p}=\frac{h}{m c^{2}}$

$$
v_{E}=\frac{r_{E}}{t_{p}}=\frac{\sqrt{2}}{16 \pi} \quad \frac{r_{1}}{R_{1}} \cdot \frac{R_{s}{ }^{2} \text {-corona }}{R^{2}} \cdot c
$$

This is the speed of the drift of the planet Earth away from the Sun Substituting the values we get $v_{E}=15.3318 \mathrm{~cm} / \mathrm{yr}$ which agrees with observed value of 15 $\mathrm{cm} / \mathrm{yr}$

$\begin{array}{ll}r & =4.14481 \times 10^{-5} \\ \mathrm{R}_{1} & =6.96 \times 10^{8} \\ \mathrm{R}_{\text {s-corona }} & =21.14 \times \mathrm{R}_{1} \\ \mathrm{R} & =149.597 \times 10^{9} \\ \mathrm{c} & =3 \times 10^{8}\end{array}$




\section{THEORY OF UNIVERSALITY}

Annexure - III

\section{Derivation of elementary charge, e:}

Assumptions:

1. Charge energy is caused by rotation of the object; and electric charge energy is equal to the rotational energy.

2. The Earth is a hollow sphere of hollowness equal to a radius of $\frac{2 R \in}{\pi}$ where $R \in$ is the radius of the Earth from the centre to the surface.

Suppose, the average mean free path of a molecule on the surface of Earth is equal to ' $\ell$ '; and the average molecular weight is $m \in$..Then, the number of free nucleons with elementary charge 'e' is : $\quad \frac{4 \pi R \epsilon^{2}}{4 \pi \ell^{2} \mathrm{~m}^{2} . .}$ ause
$m \in \ell$ is the mean free path of a free nucleon. Now, let us equate the charge energy on the surface of the Earth and the rotational energy of the Earth. Only, the surface charges contribute to the rotation of the Earth. The charges inside cancel out.

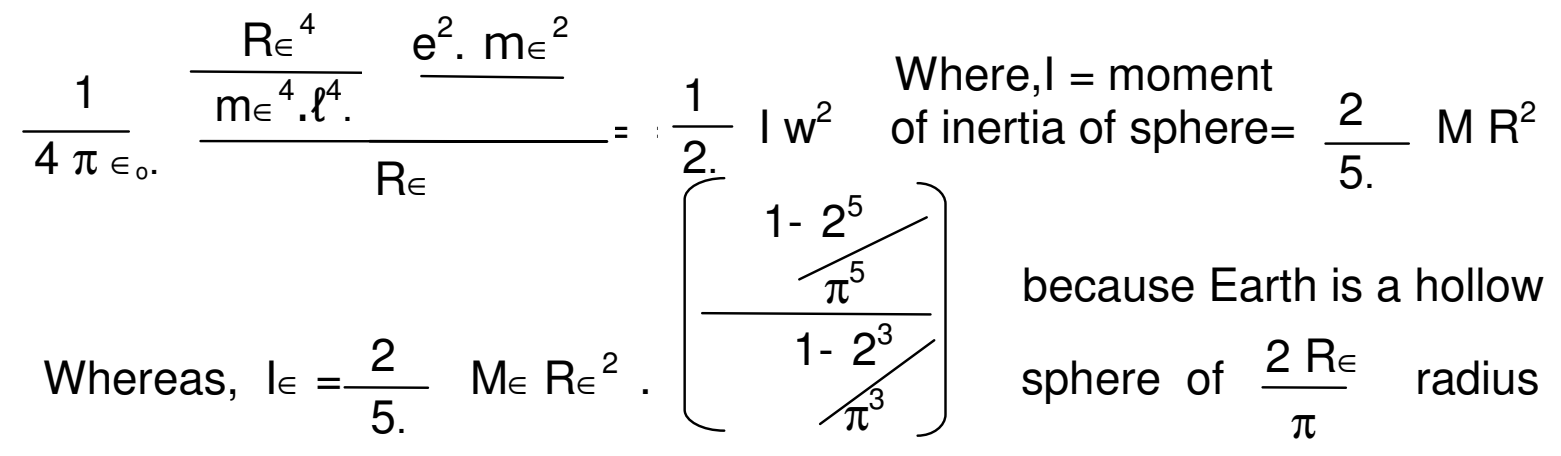
$=\frac{1}{2} \cdot \frac{2}{5 .} \cdot \frac{1}{\pi^{2}} \frac{\left(\pi^{5}-32\right)}{\left(\pi^{3}-8\right)} \mathrm{M} \in \mathrm{R} \epsilon^{2} \quad \frac{4 \pi^{2}}{\mathrm{~T}_{\epsilon}{ }^{2}}$ A

Where $M \in$ is the mass of Earth and $T_{\epsilon}$ is the period of rotation of Earth. Now, we shall derive $m \in, \ell$ and estimate the valve of ' $e$ ' theoretically. $R \in$, $M \in, T \in$ are already known. 
The Earth's surface consists of crust (with an area of 0.292 fraction of total area) and hydrosphere (with an area of 0.708 fraction of total area). The constituents

\begin{tabular}{|c|c|c|c|c|c|}
\hline of crust are: & fraction & & $\begin{array}{l}\text { mole } \\
\text { wei }\end{array}$ & & \\
\hline Oxygen & 0.4913 & $x$ & 16 & $=$ & 7.8608 \\
\hline Silicon & 0.26 & $X$ & 28 & $=$ & 7.28 \\
\hline Aluminium & 0.0745 & $X$ & 26 & $=$ & 1.937 \\
\hline Ferrous & 0.042 & $X$ & 52 & $=$ & 2.184 \\
\hline Calcium & 0.0325 & $x$ & 40 & $=$ & 1.3 \\
\hline Sodium & 0.024 & $x$ & 22 & $=$ & 0.528 \\
\hline Potassium & 0.235 & $x$ & 38 & $=$ & 0.893 \\
\hline Magnesium & 0.235 & $X$ & 24 & $=$ & 0.564 \\
\hline Hydrogen & 0.01 & $X$ & 1 & $=$ & 0.01 \\
\hline Others & 0.187 & $x$ & 20 & $=$ & 0.374 \\
\hline
\end{tabular}

The average molecular weight of the crust is $m_{1}=22.9308$ over area $a_{1}$ $=0.292$ of total area.

Similarly, Hydrosphere :

Oxygen

Hydrogen

Chlorine

Sodium

Others fraction

$\begin{array}{lll} & \text { fraction } & \\ & & \\ - & 0.8589 & \text { X } \\ - & 0.1082 & \text { X } \\ - & 0.019 & \text { X } \\ - & 0.0106 & \text { X } \\ - & 0.033 & \text { X }\end{array}$

molecular weight 
The average molecular weight $\left(m_{2}\right)$ of Hydrosphere is 15.3898 over area 0.708 of the total area.

$\mathrm{m} \in=\mathrm{a}_{1} \mathrm{~m} \in_{1}+\mathrm{a}_{2} \cdot \mathrm{m} \in_{2}=17.592$

Moreover, $\left(a_{1} \cdot l \cdot d_{1}\right) /\left(a_{2} \cdot l \cdot d_{2}\right)=\frac{m \epsilon_{1}}{m \epsilon_{2}}$

$\mathrm{d}_{2}=$ surface water density $=1025$

$d_{1}=\frac{22.9308}{15.3898} \times \frac{0.708}{0.292}=3703$

$a \cdot \ell \cdot d=a_{1} \cdot \ell \cdot d_{1}+a_{2} \cdot \ell \cdot d_{2}$

The average surface density $d=a_{1} d_{1}+a_{2} \cdot d_{2}$

$$
\Rightarrow \quad \mathrm{d}=1806.97 \text {; and } \frac{4}{3} \pi \ell^{3}=\frac{\mathrm{m} \in \mathrm{x} \mathrm{m}_{\mathrm{p}}}{\mathrm{d}} \quad \mathrm{m}_{\mathrm{p}}=\text { proton (or nucleon) }
$$

Now, $\frac{4}{3} \pi \ell^{3}=\frac{17.592 \times 1.673 \times 10^{-27}}{1806.97}$

$$
\Rightarrow \quad \ell=1.5724 \times 10^{-10}
$$

From equation $\mathrm{A}$ :

$\frac{1}{4 \pi \epsilon_{0}} \frac{\mathrm{R}^{3} \mathrm{e}^{2}}{\mathrm{~m} \epsilon^{2} \ell^{4}}=\frac{1}{2} \times 0.48263 \times \mathrm{M} \in \mathrm{R} \in^{2} \times \frac{4 \pi^{2}}{\mathrm{~T} \epsilon^{2}}$

$$
\Rightarrow \quad e^{2}=8 \pi^{3} \epsilon_{0} \quad \frac{M \in m \epsilon^{2}}{R \in} \quad x \quad \frac{\ell^{4}}{T \epsilon^{2}} \quad \times 0.48263
$$

Substituting the values:

$$
\begin{array}{ll}
\epsilon_{\circ}=8.85 \times 10^{-12} & \mathrm{R} \in=6.371 \times 10^{6} \\
\mathrm{M} \in=5.977 \times 10^{24} & \mathrm{~T} \in=2.393 \times 3.6 \times 10^{4} \\
\mathrm{~m} \in=17.592 & \ell=1.5724 \times 10^{-10}
\end{array}
$$

We get

$\mathrm{e}^{2}=2.5368603 \times 10^{-38}$

$\begin{aligned}= & =1.592752 \times 10^{-19} \\ & 1.602 \times 10^{-19}\end{aligned}$ which agrees with the experimental value of

The slight discrepancy i.e., $0.925 \%$ is due to approximation in $m \in$ and $\ell$. Hence, the assumptions are correct and valid. 


\section{PREDICTION - 3 :}

In the Solar System, the ratio of the mass of Sun to the mass of Saturn is nearly same as the ratio of the mass of one neutron plus one proton to the mass of an electron. We can predict that the Solar System follows Deuterium atomic model with the Sun as the nucleus and Saturn as the electron. Moreover, we can also predict that Saturn is the only planet which has come out of Sun and all other planets of the Solar System are like sub-atomic particles of the Deuterium atomic model.

\section{PREDICTION - 4 :}

We have seen in PREDICTION - 1 that the force of Sun on Earth is nearly 20,000 billion times less than the force of Earth on the Sun. We can predict that the Solar System is a whirlpool of space in which all the planets and the Sun are floating and the planets are kept in motion by the space in motion and the Sun happens to be at the centre and the Sun's hold on the planets is feeble; compared to the planets' force on the Sun. Moreover, we can also predict that the planets like Earth can become stars after it slows down to a speed of ' $c$ ' from its present speed of $c+v_{1}$

\section{PREDICTION - 5 :}

The central black hole is matter with a highest speed of ' $c$ '; and all the protons in the universe rotate at nearly the speed of light ' $c$ ' in the direction of the central black hole; and all the electrons in the universe rotate in the opposite direction at nearly the speed ' $c$ '.

\section{PREDICTION - 6 :}

A freely moving sphere in space will have a rotational motion also, if it has a linear motion, and the corresponding relationship between the rotational speed and the linear speed can be derived.

\section{PREDICTION - 7 :}

The central body of the universe is rotating in one direction; and all the protons of the universe are rotating at nearly the speed of light ' $c$ ' in that direction; electrons in the opposite direction at nearly the speed of light ' $c$ '. 


\section{PREDICTION - 8 :}

The elementary particles neutron, proton and electron are stable because the universe is vibrating at the speed of light ' $c$ ' along with the particles which also vibrate at the speed of light ' $c$ '.

\section{PREDICTION - 9 :}

The least speed of any celestial body in absolute sense; outside the central body is ' $c$ '; the highest speed within the central body is ' $c$ '.

\section{PREDICTION - 10 :}

The celestial objects jet out like stream of particles from the central body. The central body and the celestial objects rotate in the same direction and the celestial objects go around the central body in the same direction.

\section{PREDICTION - 11 :}

Positive and Negative electrical charges are because of rotation of protons and electrons at nearly the speed ' $c$ ' in opposite directions.

\section{PREDICTION - 12 :}

In reflection, refraction and diffraction of light; light is attracted by the nucleus of protons and neutrons ( which behave as matter) and repelled by electrons ( which behave as anti-matter ); because light behaves like matter particles.

\section{PREDICTION - 13 :}

The universe is a finite sphere with space, celestial objects, energy and expanding. It appears to be infinite and every point of the universe appears to be a centre ; because, of multiple reflections of light and energy by the surface ( or boundary or edge which is actually expanding ) back into the universe.

\section{PREDICTION - 14 :}

The universe is expanding into void and will fall back into the central black hole; because of all anti-matter getting exploded into matter or space which will then be attracted by the matter in the central black hole. 


\section{PREDICTION - 15 :}

The plane of the planetary orbits around the sun is inclined in the direction of movement of the solar system in Milky way galaxy and the planets are partly dragging the Sun in its motion around the galaxy; along with space. This is so because; something has to energize the movement of sun against spatial friction. Space in motion also causes partly the movement of the solar system in the galaxy.

\section{SNELL's Law :}

The coefficient of refractive indices:

Light behaves as matter waves; and is repelled by electrons in medium which behave as anti-matter; and attracted by neutrons / protons which are matter which causes refraction. Suppose, light has a mass $m_{1}$ and $m_{2}$; and speeds $\mathrm{c}_{1}$ and $\mathrm{c}_{2}$ in the two media. The horizontal component of the force

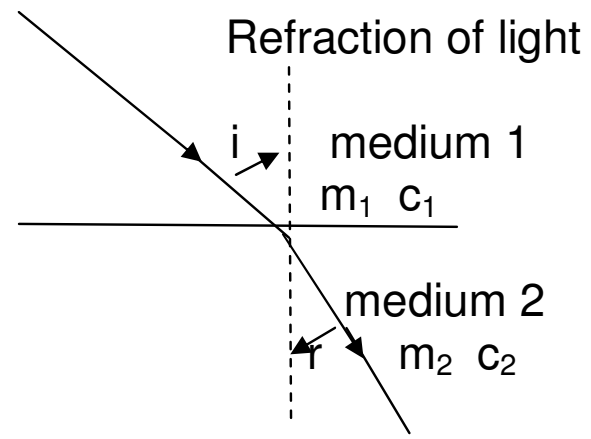
of light from medium 1 to medium 2 does not change; as there is no force in this direction.

Therefore,

$$
\begin{aligned}
& \frac{\mathrm{m}_{1}{ }^{2} \mathrm{c}_{1}{ }^{3}}{\mathrm{~h}} \sin \mathrm{i}=\frac{\mathrm{m}_{2}{ }^{2} \mathrm{c}_{2}{ }^{3}}{\mathrm{~h}} \sin \mathrm{r} \text { as per my theory of universality. } \\
& \Rightarrow \frac{\sin \mathrm{i}}{\sin \mathrm{r}}=\mathrm{n}=\frac{\mathrm{m}_{2}{ }^{2} \mathrm{c}_{2}{ }^{3}}{\mathrm{~m}_{1}{ }^{2} \mathrm{c}_{1}{ }^{3}}=\frac{\mathrm{m}_{2}{ }^{2} \mathrm{c}_{2}{ }^{4}}{\mathrm{~m}_{1}{ }^{2} \mathrm{c}_{1}{ }^{4}} \frac{\mathrm{c}_{1}}{\mathrm{c}_{2}}
\end{aligned}
$$

Now energy is conserved $\Rightarrow m_{1} c_{1}{ }^{2}=m_{2} c_{2}{ }^{2}$

$$
\mathrm{n}=\frac{\sin \mathrm{i}}{\sin \mathrm{r}}=\frac{\mathrm{c}_{1}}{\mathrm{c}_{2}}=\text { coefficient of refraction }
$$

Hence, Snell's law is derived as per theory. 
3.GRAVITATIONAL LAW: $F=G \frac{M_{1} M_{2}}{R^{2}}$

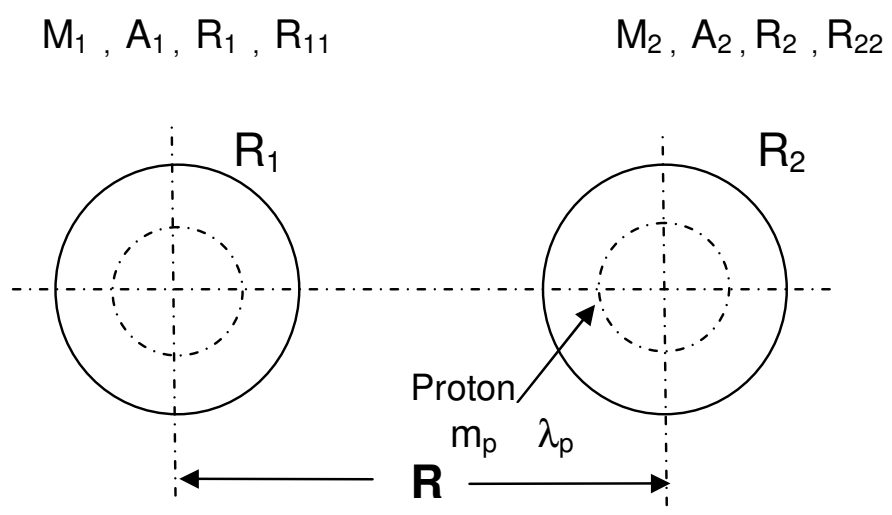

Take two bodies of mass $M_{1}, M_{2}$; radii $R_{1}, R_{2}$; mean free path of molecules $r_{1}, r_{2}$ molecular weight $z_{1}, z_{2}$; and having $A_{1}, A_{2}$ moles of weight. Consider one mole of each with radii $R_{11}, R_{22}$.

As per my theory, the force due to one mole of $M_{1}$ at a distance of $R$ is: $\frac{1}{2} \quad \frac{4 \pi R^{2}{ }_{11}}{4 \pi r_{1}{ }^{2}} \quad \frac{z_{1} m^{2} c^{3}}{h} \quad \frac{R^{2}{ }_{22}}{R^{2}}$ on one mole of $M_{2}$

The force will act on one proton along the line joining the centers of mass. Hence, the force on one mole of $\mathrm{M}_{2}$ along the line joining

centers of mass is: $\quad \frac{1}{2} \frac{z_{1} R^{2}{ }_{11}}{r_{1}{ }^{2}} \frac{m^{2} c^{3} R^{2}}{h R^{2}} \frac{m_{p}}{z_{2} N_{a} m}$ where $\mathrm{N}_{\mathrm{a}}$ is Avogadro constant and $\mathrm{N}_{\mathrm{a}} \cdot \mathrm{m}=1$.

The force due to $A_{1}$ moles of $M_{1}$ on $A_{2}$ moles of $M_{2}$ along or parallel to the lines joining the centers of mass on the nearest protons would be;

$$
F_{12}=\frac{1}{2} \frac{R_{11}^{2}}{r_{1}{ }^{2}} \quad \frac{z_{1} A_{1} m^{2} c^{3}}{h} \frac{R_{22}^{2}}{r_{2}^{2}} \frac{r_{2}{ }^{2}}{R^{2}} \frac{A_{2} m_{p}}{z_{2.1}}
$$

we know that $r_{2}=z_{2} \cdot\left(2 \lambda_{p}\right) \quad\left\{\right.$ mean free path of proton=2 $\left.\lambda_{p}\right\}$

Also, $A_{1} z_{1} m=\frac{M_{1}}{N_{a}}$; and $A_{2} z_{2} m=\frac{M_{2}}{N_{a}}$

Therefore ,

$$
F_{12}=\frac{1}{2} \frac{R^{2}{ }_{11}}{r_{1}{ }^{2}} \quad \frac{M_{1} M_{2}}{N_{a}{ }^{2}} \quad \frac{c^{3}}{h} \frac{R^{2}{ }_{22}}{r_{2}{ }^{2}} \quad \frac{z_{2}{ }^{2} 4 \lambda_{p}{ }^{2}}{z_{2}} m_{p}
$$


Moreover, for one mole of a body $\frac{z_{1} m N_{a}}{\frac{4 \pi R^{3}}{3}}=\frac{z_{1} m}{\frac{4}{3} \pi r_{1}^{3}}$

$$
\Rightarrow \frac{\mathrm{R}_{11}^{3}}{\mathrm{r}_{1}^{3}}=\mathrm{N}_{\mathrm{a}} \Rightarrow \frac{\mathrm{R}_{22}^{3}}{\mathrm{r}_{2}^{3}}=\mathrm{N}_{\mathrm{a}}
$$

from $A$ and $B$, we have

$F_{12}=\frac{1}{2} \cdot \frac{N_{a}^{2 / 3} \cdot N_{a}^{2 / 3}}{N_{a}^{2}} \cdot 4 \cdot \frac{M_{1} M_{2}}{R^{2}} \cdot \frac{c^{3} \cdot \lambda_{p}{ }^{2} \cdot m_{p}}{h}$

Similarly, the force of $M_{2}$ on $M_{1}$ will be an equal amount. The total force of gravitation between two bodies of mass $M_{1}$ and $M_{2}$ along the line joining the centers of mass at a distance $R$ is :

$F=F_{21}+F_{12}=\frac{4 c^{3} \cdot \lambda_{p}^{2} \cdot m_{p}}{N_{a}^{2 / 3} \cdot h} \quad \frac{M_{1} M_{2}}{R^{2}}$

Hence, we can conclude that

$G=\frac{4 c^{3} \cdot \lambda_{p}^{2} \cdot m_{p}}{N_{a}^{2 / 3} h}$

works out to be $\mathrm{G}=6.66889 \times 10^{-11}$ and agrees with experimental

value of $\mathrm{G}=6.67384 \times 10^{-11}$

Hence Newton's law of gravitation for large distances is derived as per my theory of universality

\section{PREDICTION - 16 :}

4.The surface speed of a charged fundamental particle : In the case of charged fundamental particles like, proton and electron charge energy is equal to the mass energy as per observation and also equal to the rotational energy.

That is $m_{0} c^{2}=\frac{1}{2} \cdot \frac{2}{5} \cdot m^{2} \cdot w^{2}=\frac{1}{2} m v^{2} \cdot \frac{2}{5}=\frac{1}{5} m v^{2}$

where $v$ is the surface speed of the particle of rotation

$$
\Rightarrow \frac{1}{5} \frac{m_{0}}{\sqrt{\frac{1-v^{2}}{c^{2}}}} \cdot v^{2}=m_{0} c^{2}
$$




$$
\begin{aligned}
& \Rightarrow \quad v^{4}+25 c^{2} v^{2}-25 c^{4}=0 \\
& \Rightarrow \quad v^{2}=\frac{(-25 \pm 26.926) c^{2}}{2} \\
& \Rightarrow \quad v=0.98133 . c \text {, or } \sqrt{-25.963 . c}
\end{aligned}
$$

That is to say the charged positive matter particle i.e,. proton, moves with a surface speed of rotation $0.98133 \mathrm{c}$ in the direction of the central black hole which gives it the positive charge; and since, the speed is less than ' $c$ ' it is matter particle.

The charged negative anti-matter particle, i.e,. electron, moves with a surface speed of rotation of $\sqrt{-25.963} \mathrm{c}$ in the direction opposite that of the central black hole which gives it the negative charge; and since, the speed is more than ' $c$ ' it is anti-matter particle.

5.Surface density of central black hole $(\rho \mathrm{BH})$ : Compton wave length of Sun $=2 \pi \cdot R_{\mathrm{sc}}$ where $R_{\mathrm{sc}}$ is the solar corona radius; and Sun is anti-matter The central black hole keeps a matter equivalent mass of every anti-matter particle / object jetting out of it ; whose mass and size keep changing as per the antimatter object which came out. However, the surface density of the central black hole remains constant.

The mass of the matter particle equivalent of this Compton wave length of Sun (on the central black hole) $=\frac{h}{2 \pi . R_{s c}}$ and the Compton wave length of the matter particle equivalent of the Sun (on the central black hole is $\frac{h}{M_{s} C}$ Therefore, the surface density of the central black hole is

$$
\begin{aligned}
& \rho \mathrm{BH}=\left[\frac{\mathrm{h}}{2 \pi \cdot R_{\mathrm{sc}} \mathrm{c}}\right]^{/} \frac{4 \pi .}{3} \quad\left[\frac{\mathrm{h}}{2 \pi \cdot \mathrm{M}_{\mathrm{s}} \mathrm{c}}\right]^{3} \\
& \mathrm{\rho BH}=3 \pi . \frac{\mathrm{M}_{\mathrm{s}}{ }^{3} \cdot \mathrm{c}^{2}}{\mathrm{R}_{\mathrm{sc} \cdot \mathrm{h}^{2}}}
\end{aligned}
$$

Under normal circumstances, the surface density of the central black hole is constant in equilibrium with the universe. Hence, $M_{s}{ }^{3} \propto R_{s c}$.

That is, the cube of mass of a star is directly proportional to the star's corona radius. 


\section{THEORY OF UNIVERSALITY}

This theory is an attempt to describe the universal phenomena like space, time, matter and energy as an inter-relationship bound by a newly discovered force named as the universal force. The universal force is shown to be the force of gravitation, electricity, magnetism, strong nuclear and weak nuclear forces. I believe any other force, hitherto fore not discovered; also, can be explained in terms of this universal force.

Liberal use of wave-particle duality, relativity, quantum concepts is made to achieve a harmonious and comprehensive synthesis of all the existing beliefs in physics into a new theory with some new concepts added here and there. While adding new concepts, enormous care has been taken to ensure that the existing beliefs are not contradicted. Moreover, the new concepts are proved to be correct theoretically by deriving the constants like $\mathrm{G}, \sigma$, etc.,

I believe, we are still at the beginning of civilization. The number of exciting possibilities in the progress of humankind and survival of the planet seem to be amazing. I have laid the foundation for the modern physics with this theory of universality. Further development in understanding the concepts and the phenomena of the universe seems to be unlimited.

(Kasibhatla Surya Narayana)

Address :

\section{K.S. Narayana}

E-002, E-Block

Terrace Garden Apartments

Itamadu Main Road, BSK - III stage

Bangalore - 560085, INDIA

$\mathrm{Ph}:+91-080-26421544(\mathrm{R})$

+91-080-66626963(O)

E : ksuraxn@yahoo.co.in 


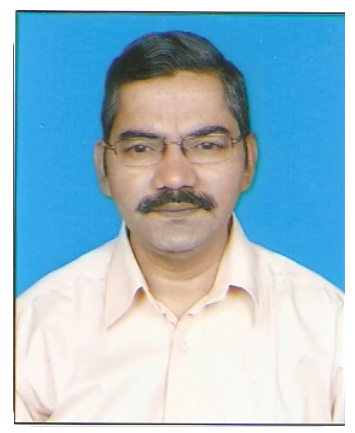

K.S. Narayana

(KASIBHATLA SURYA NARAYANA )(1959-)

Author : Theory of Universality(1982 - )

The author is a graduate in Electronics from Indian Institute of

Technology (1977-82);

and has passed the Civil Services (Main) Exam , 1983.

He learned Physics during his graduate studies in the institute. He has worked in Computer Application Software since 1982, and is presently working for the Progress Department of HMT Machine Tools Ltd., Bangalore Complex, BANGALORE, India.

With an interest in developing a fundamental theory in Physics; which will encompass the existing beliefs; and solve some of the paradoxes already existing since 1960s, he has taken up this Theory of Universality as a project out of personal interest and hobby. He is not basically a physicist by career, however, with whatever knowledge picked up during the graduate studies, and after consulting some periodicals; he went on to develop this theory.

This theory will serve to be the basic foundation for the future development of Modern Physics; and also be the GUT ( Grand Unified Theory ) in Physics ; unifying the four fundamental quantities space, time, matter and energy; and the four fundamental forces gravitation, electromagnetism, strong and weak nuclear forces.

It is sincerely hoped that this theory will generate interest in the reading public and become useful in further understanding of universe and universality.

\section{K.S. Narayana}




\section{ACKNOWLEDGEMENT}

1. Prof. R. Chandramani, Professor of Physics, Department of Physics, Dayanand Sagar College of Engineering, Bangalore University, Bangalore, India : for her studious efforts in going through the theory and suggestions.

\section{REFERENCES}

[1]. David Halliday, Robert Resnick, Jearl Walker : Fundamentals of Physics Extended, $8^{\text {th }}$ Edition. (Book)

[2]. Raymond A. Serway, John W. Jewett : Physics for Scientists and Engineers with Modern Physics. ( Book )

[3]. Raymond A. Serway, Clement J. Moses, Curt A. Moyer : Modern Physics. ( Book )

[4]. B.V. Rama Rao : A Text Book of Modern Physics

[5]. Orient Longman : (C) Oliver \& Boyd 1971 : Clark's Tables Science Data Book 